\title{
The Cosmic Ultraviolet Baryon Survey (CUBS) I. Overview and the diverse environments of Lyman limit systems at $z<1^{\star}$
}

Hsiao-Wen Chen ${ }^{1} \dagger$, Fakhri S. Zahedy ${ }^{2}$, Erin Boettcher ${ }^{1}$, Thomas M. Cooper ${ }^{2}$, Sean D. Johnson ${ }^{2,3} \ddagger$, Gwen C. Rudie ${ }^{2}$, Mandy C. Chen ${ }^{1}$, Gregory L. Walth ${ }^{2}$, Sebastiano Cantalupo ${ }^{4}$, Kathy L. Cooksey ${ }^{5}$, Claude-André Faucher-Giguère ${ }^{6}$, Jenny E. Greene ${ }^{3}$, Sebastian Lopez ${ }^{7}$, John S. Mulchaey ${ }^{2}$, Steven V. Penton ${ }^{8}$, Patrick Petitjean $^{9}$, Mary E. Putman ${ }^{10}$, Marc Rafelski ${ }^{11,12}$, Michael Rauch ${ }^{2}$, Joop Schaye ${ }^{13}$, Robert A. Simcoe ${ }^{14}$, and Benjamin J. Weiner ${ }^{15}$

${ }^{1}$ Department of Astronomy \& Astrophysics, The University of Chicago, Chicago, IL 60637, USA

${ }^{2}$ The Observatories of the Carnegie Institution for Science, 813 Santa Barbara Street, Pasadena, CA 91101, USA

${ }^{3}$ Department of Astrophysics, Princeton University, Princeton, NJ 08544, USA

${ }^{4}$ Department of Physics, ETH Wolfgang-Pauli-Strasse 27, 8093, CH-8093 Zürich, Switzerland

${ }^{5}$ Department of Physics and Astronomy, University of Hawai'i at Hilo, Hilo, HI 96720, USA

${ }^{6}$ Department of Physics $\mathscr{G}$ Astronomy and Center for Interdisciplinary Exploration and Research in Astrophysics (CIERA), Northwestern University, 1800 Sherman Ave, Evanston, IL 60201, USA

${ }^{7}$ Departamento de Astronomía, Universidad de Chile, Casilla 36-D, Santiago, Chile

${ }^{8}$ Laboratory For Atmospheric and Space Physics, University of Colorado, Boulder, CO 80303, USA

${ }^{9}$ Institut dAstrophysique de Paris, CNRS-SU, UMR 7095, 98bis bd Arago, Paris F-75014, France

${ }^{10}$ Department of Astronomy, Columbia University, New York, NY 10027, USA

${ }^{11}$ Space Telescope Science Institute, Baltimore, MD 21218, USA

${ }^{12}$ Department of Physics \& Astronomy, Johns Hopkins University, Baltimore, MD 21218, USA

${ }^{13}$ Leiden Observatory, Leiden University, PO Box 9513, NL-2300 RA Leiden, the Netherlands

${ }^{14}$ MIT-Kavli Institute for Astrophysics and Space Research; 77 Massachusetts Ave., Cambridge, MA 02139, USA

${ }^{15}$ Steward Observatory, University of Arizona, Tucson, AZ 85721, USA

\begin{abstract}
We present initial results from the Cosmic Ultraviolet Baryon Survey (CUBS). CUBS is designed to map diffuse baryonic structures at redshift $z \lesssim 1$ using absorptionline spectroscopy of $15 \mathrm{UV}$-bright QSOs with matching deep galaxy survey data. CUBS QSOs are selected based on their NUV brightness to avoid biases against the presence of intervening Lyman Limit Systems (LLSs) at $z_{\text {abs }}<1$. We report five new LLSs of $\log N\left(\mathrm{H}_{\mathrm{I}}\right) / \mathrm{cm}^{-2} \gtrsim 17.2$ over a total redshift survey pathlength of $\Delta z_{L L}=9.3$, and a number density of $n(z)=0.43_{-0.18}^{+0.26}$. Considering all absorbers with $\log N\left(\mathrm{H}_{\mathrm{I}}\right) / \mathrm{cm}^{-2}>16.5$ leads to $n(z)=1.08_{-0.25}^{+0.31}$ at $z_{\text {abs }}<1$. All LLSs exhibit a multi-component structure and associated metal transitions from multiple ionization states such as $\mathrm{C}_{\text {II }}, \mathrm{C}_{\text {III, }} \mathrm{Mg}$ II, $\mathrm{Si}$ II, $\mathrm{Si}$ III, and $\mathrm{O}_{\text {vi absorption. Differential chemical }}$ enrichment levels as well as ionization states are directly observed across individual components in three LLSs. We present deep galaxy survey data obtained using the VLT-MUSE integral field spectrograph and the Magellan Telescopes, reaching sensitivities necessary for detecting galaxies fainter than $0.1 L_{*}$ at $d \lesssim 300$ physical kpc (pkpc) in all five fields. A diverse range of galaxy properties is seen around these LLSs, from a low-mass dwarf galaxy pair, a co-rotating gaseous halo/disk, a star-forming galaxy, a massive quiescent galaxy, to a galaxy group. The closest galaxies have projected distances ranging from $d=15$ to $72 \mathrm{pkpc}$ and intrinsic luminosities from $\approx 0.01 L_{*}$ to $\approx 3 L_{*}$. Our study shows that LLSs originate in a variety of galaxy environments and trace gaseous structures with a broad range of metallicities.
\end{abstract}

Key words: surveys - galaxies: haloes - quasars: absorption lines 


\section{INTRODUCTION}

The circumgalactic medium (CGM) and intergalactic medium (IGM) contain fuel for future star formation and a record of past feedback. They are uniquely sensitive to the physics of baryonic flows - one of the principal missing ingredients in our understanding of galaxy evolution (for reviews see e.g., Somerville \& Davé 2015; Naab \& Ostriker 2017; Tumlinson et al. 2017). While QSO absorption spectroscopy provides a powerful tool for probing the diffuse gas phase in intergalactic and circumgalactic space, a comprehensive study of the CGM requires matching galaxy survey data. Previous joint galaxy and absorber studies have focused primarily on two disjoint epochs, $z<0.4$ (e.g., Chen 2017; Kacprzak 2017; Tumlinson et al. 2017 for recent reviews) and $z \approx 2$ (e.g., Steidel et al. 2010; Rudie et al. 2012; Turner et al. 2014; Rudie et al. 2019). However, as the cosmic star formation rate density (SFRD) declines rapidly from $z \approx 1.5$ to the present day, the CGM remains poorly constrained over a significant fraction of cosmic history (see Burchett et al. 2019 for recent effort in probing the warmhot CGM through observations of Ne viII absorption).

Observations of the co-evolution of galaxies with their surrounding gas complements the progress both in wide-field galaxy surveys and in theoretical models of how galaxies form and evolve. In particular, state-of-the-art cosmological simulations, incorporating realistic star-formation and feedback recipes, can both match the large-scale statistical properties of galaxies and reproduce the observed small-scale features (e.g., Vogelsberger et al. 2014; Hopkins et al. 2014; Schaye et al. 2015; Wang et al. 2015; Dubois et al. 2016). But these models have fallen short in simultaneously matching the spatial profiles of a wide range of heavy ions (such as $\mathrm{Mg}^{+}, \mathrm{C}^{3+}, \mathrm{O}^{5+}$ ) observed in the CGM where the majority of the baryons reside (e.g., Hummels et al. 2013; Liang et al. 2016; Oppenheimer et al. 2016; Nelson et al. 2018; Ji et al. 2019). This mismatch suggests that our understanding of the nature and effects of gas inflows and outflows is still incomplete. Identifying the missing physics that governs the dynamical state of the CGM provides an unparalleled constraint on the manner and mode of feedback in galaxies.

To enable systematic studies of the diffuse CGM and IGM, we are conducting the Cosmic Ultraviolet Baryon Survey (CUBS), which is a large Hubble Space Telescope (HST) Cycle 25 General Observer Program (GO-CUBS; PID $=15163$; PI: Chen). It is designed to map the diffuse baryonic structures at $z \lesssim 1$, using absorption-line spectroscopy of $15 \mathrm{UV}$ bright QSOs with matching deep galaxy survey data. The primary goal of CUBS is to establish a legacy galaxy and absorber sample to enable systematic studies of the co-evolution of galaxies and their surrounding diffuse gas at a time when the SFRD undergoes its most dramatic changes, thereby gaining key insights into how galaxy growth is regulated by accretion and outflows. The CUBS program exploits the synergy between space-based UV spec-

Paranal Observatory, and the NASA/ESA Hubble Space Telescope operated by the Space Telescope Science Institute and the Association of Universities for Research in Astronomy, Inc., under NASA contract NAS 5-26555.

† E-mail: hchen@oddjob.uchicago.edu

$\ddagger$ Carnegie-Princeton Fellow troscopy and ground-based wide-field surveys, as well as optical echelle spectroscopy, for advancing a comprehensive understanding of the cosmic evolution of baryonic structures.

Here we present initial results from the CUBS program, reporting five new Lyman limit systems (LLSs) discovered at $z<1$ along the CUBS QSO sightlines. In addition, we present the galactic environment of these LLSs uncovered from an ongoing galaxy survey in the CUBS fields, using the Magellan Telescopes and the VLT Multi-Unit Spectroscopic Explorer (MUSE; Bacon et al. 2010). LLSs arise in optically thick gas with opacity $\tau_{912} \gtrsim 1$ to ionizing photons at restframe wavelength $\approx 912 \AA$ (or equivalently neutral hydrogen column density $\left.\log N\left(\mathrm{H}_{\mathrm{I}}\right) / \mathrm{cm}^{-2} \gtrsim 17.2\right)$. On cosmological scales, the incidence of these optically-thick absorbers determines the mean free path of ionizing photons and serves as a key ingredient for computing the photoionization rate in the IGM (e.g., Rudie et al. 2013; Faucher-Giguère 2020). In individual galactic halos, these absorbers are commonly seen

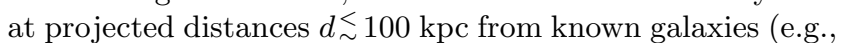
Chen et al. 1998, 2001; Rudie et al. 2012; Thom et al. 2012; Werk et al. 2014; Johnson et al. 2015; Prochaska et al. 2017) with a mean covering fraction of $\kappa_{\tau_{912} \geqslant 1}(d<100 \mathrm{kpc}) \geq 70 \%$ (e.g., Chen et al. 2018). The large scatter observed in both gas density and metallicity of the absorbing gas (e.g., Zahedy et al. 2019; Lehner et al. 2019) makes these absorbers a promising signpost of either infalling clouds (e.g., Maller \& Bullock 2004; Faucher-Giguère \& Kereš 2011; Fumagalli et al. 2011; van de Voort et al. 2012; Afruni et al. 2019) or outflows (e.g., Faucher-Giguère et al. 2015, 2016) in galactic halos, or a combination thereof (e.g., Hafen et al. 2017). We examine these different scenarios based on the galaxy environment revealed in the accompanying galaxy survey data.

The paper is organized as follows. In Section 2, we describe the design of the CUBS program and related spectroscopic observations. We describe the search and identification of LLSs along the CUBS QSO sightlines in Section 3 , and their galactic environments in Section 4. In Section 5 , we discuss the implications of our findings. Throughout the paper, we adopt a standard $\Lambda$ cosmology, $\Omega_{M}=0.3$ and $\Omega_{\Lambda}=0.7$ with a Hubble constant $H_{0}=70 \mathrm{~km} \mathrm{~s}^{-1} \mathrm{Mpc}^{-1}$.

\section{THE CUBS PROGRAM}

The CUBS program is designed to map the dominant cosmic baryon reservoirs in intergalactic and circumgalactic space at intermediate redshifts using QSO absorption spectroscopy, bridging the gap between existing efforts at $z<0.4$ and at $z \approx 2$. It utilizes the high UV throughput and medium spectral resolution offered by the Cosmic Origins Spectrograph (COS; Green et al. 2012) on board HST for probing the physical conditions and chemical content of diffuse gas based on observations of a suite of absorption transitions from different ions. Specifically, COS with the G130M and G160M gratings provides spectral coverage over $\lambda=1100-1800 \AA$ for a wide range of ionic transitions at $z<1$. These include the hydrogen Lyman-series transitions and heavy elements such as carbon, nitrogen, oxygen, silicon, sulfur, etc. in several ionization states (examples are presented in Boettcher et al. 2020, Cooper et al. 2020, Johnson et al. 2020, and Zahedy et al. 2020 in preparation). An added bonus of targeting the redshift range at $z>0.4$ is 
the ability to precisely measure the neutral hydrogen column density, $N(\mathrm{HI})$, using the higher-order Lyman series absorption transitions (e.g., Rudie et al. 2013; Chen et al. 2018; Zahedy et al. 2019).

In addition, a critical component of the CUBS program is a comprehensive deep galaxy survey in the fields around these 15 QSOs. The locations and properties of galaxies, together with the absorption properties of associated halos from the COS spectra, provide direct constraints for feeding and feedback in galactic halos. Our galaxy survey is carried out both in space using the Wide Field Camera 3 (WFC3) and the IR channel in parallel slitless grism mode, and on the ground using the VLT and Magellan telescopes. The slitless grism spectroscopy utilizes the G102 and G141 grisms to target nebular lines such as [O III], $\mathrm{H} \beta$, and $\mathrm{H} \alpha$ at $z \lesssim 1$ from galaxies as faint as $A B(H) \approx 23-24$ mag. While the field will be offset by 3-4 Mpc from the QSO, it complements the ground-based galaxy survey for mapping the large-scale structures in the QSO fields.

The ground-based galaxy survey consists of three components: (1) a shallow and wide component using the IMACS multi-object imaging spectrograph (Dressler et al. 2006) on the Magellan Baade telescope to target $L_{*}$ galaxies at $z_{\text {gal }} \lesssim 0.8$ and angular distances at $\theta \lesssim 10^{\prime}$ (corresponding to $d<3-5$ physical Mpc) from the QSO sightline, (2) a deep and narrow component using the Low Dispersion Survey Spectrograph 3 (LDSS3) on the Magellan Clay telescope to target all galaxies as faint as $0.1 L_{*}$ up to $z \approx 1$ (and fainter at lower redshifts) at $\lesssim 3^{\prime}$ in angular radius (corresponding to $d<300-500$ physical kpc; pkpc) from the QSO sightline, and (3) an ultradeep component using MUSE on the VLT UT4 to target galaxies as faint as $\approx 0.01 L_{*}$ at $z \approx 1$ at $\lesssim 30^{\prime \prime}$ in angular radius (corresponding to $d<250$ physical kpc) from the QSO sightline. The shallow and wide component will enable large-scale $(\approx 1-10 \mathrm{Mpc})$ cross-correlation studies between gas and galaxies. The ultradeep and deep and narrow components enable detailed studies of gas flows and the chemical enrichment in the CGM at projected distances $d \lesssim 300$ pkpc from galaxies with mass as low as $M_{\text {star }} \sim 10^{9} \mathrm{M}_{\odot}$ at $z \lesssim 1$. The primary scientific objectives are: (1) to measure the cosmic mass density evolution of heavy ions; (2) to determine the metallicity and ionization state of the diffuse CGM and IGM; (3) to constrain the origin and evolution of the chemically-enriched CGM in halos of different masses and star formation histories; and (4) to investigate the environmental effects in distributing heavy elements beyond galaxy halos. Here we describe the program design and associated spectroscopic observations.

\subsection{Program Design}

To facilitate a systematic and unbiased study of the CGM/IGM at $z \approx 0.4-1$, the CUBS QSOs are selected to be at $z_{\mathrm{QSO}} \approx 0.8$ and bright in the GALEX near-UV bandpass (NUV; 1770-2730 ^). The QSOs are selected from existing surveys with available spectra for redshift confirmations, including the Hamburg/ESO survey (Wisotzki et al. 2000), the Sloan Digital Sky Survey (SDSS; York et al. 2000; Eisenstein et al. 2011), the Ultraviolet-bright Quasar Survey (UVQS; Monroe et al. 2016), and our own spectroscopic observations for confirmations. Redshift uncertainties are typically $d z /(1+z) \approx 0.002$ for the UVQS and significantly better
Table 1. Summary of the CUBS QSO Sample

\begin{tabular}{ccccccc}
\hline \hline & & & & FUV & NUV & \\
QSO & RA(J2000) & Dec(J2000) & $z_{\text {QSO }}$ & (mag) & (mag) & Ref. $^{a}$ \\
\hline J0028-3305 & $00: 28: 30.405$ & $-33: 05: 49.25$ & 0.887 & 17.33 & 16.52 & $(1)$ \\
J0110-1648 & $01: 10: 35.511$ & $-16: 48: 27.70$ & 0.777 & 17.31 & 16.72 & $(2)$ \\
J0111-0316 & $01: 11: 39.171$ & $-03: 16: 10.89$ & 1.234 & 18.47 & 16.66 & $(3)$ \\
J0114-4129 & $01: 14: 22.123$ & $-41: 29: 47.29$ & 1.018 & 18.33 & 16.71 & $(4)$ \\
J0119-2010 & $01: 19: 56.091$ & $-20: 10: 22.73$ & 0.812 & 16.86 & 16.15 & $(5)$ \\
J0154-0712 & $01: 54: 54.682$ & $-07: 12: 22.17$ & 1.289 & 17.07 & 16.40 & $(3)$ \\
J0248-4048 & $02: 48: 06.286$ & $-40: 48: 33.66$ & 0.883 & 16.11 & 15.47 & $(4)$ \\
J0333-4102 & $03: 33: 07.076$ & $-41: 02: 01.15$ & 1.124 & 17.60 & 16.33 & $(4)$ \\
J0357-4812 & $03: 57: 21.918$ & $-48: 12: 15.16$ & 1.016 & 17.76 & 16.84 & $(6)$ \\
J0420-5650 & $04: 20: 53.907$ & $-56: 50: 43.96$ & 0.944 & 17.61 & 16.86 & $(4)$ \\
J0454-6116 & $04: 54: 15.952$ & $-61: 16: 26.56$ & 0.784 & 16.89 & 16.16 & $(3)$ \\
J2135-5316 & $21: 35: 53.202$ & $-53: 16: 55.82$ & 0.806 & 17.13 & 15.94 & $(3)$ \\
J2308-5258 & $23: 08: 37.796$ & $-52: 58: 48.94$ & 1.067 & 17.97 & 16.73 & $(4)$ \\
J2339-5523 & $23: 39: 13.218$ & $-55: 23: 50.84$ & 1.354 & 17.91 & 16.37 & $(4)$ \\
J2245-4931 & $22: 45: 00.207$ & $-49: 31: 48.46$ & 1.003 & 18.10 & 16.90 & $(3)$ \\
\hline
\end{tabular}

${ }^{a}$ Referenes: (1) Lamontagne et al. (2000); (2) Perlman et al. (1998);

(3) Monroe et al. (2016); (4) Wisotzki et al. (2000); (5) Jones et al.

(2009); (6) Savage et al. (1978).

for SDSS (e.g., Hewett \& Wild 2010). By targeting QSOs at $z_{\mathrm{QSO}}=0.8-1.3$, we optimize the survey efficiency and maximize the survey pathlength offered by the spectral coverage of COS for each QSO sightline.

The NUV magnitude limited QSO selection criterion is motivated by the expectation that the presence of a LLS or a pLLS at $z \lesssim 0.9$ attenuates the background QSO light in the far-UV channel (FUV; 1350-1780 A; see the top panel of Figure 1). Consequently, targeting known FUV-bright QSOs at $z_{\mathrm{QSO}} \gtrsim 0.8$ would impose a bias against sightlines intercepting a LLS or partial LLS at lower redshifts. Finally, we select the QSOs from regions covered by the Dark Energy Survey $^{1}$ (DES; e.g., Drlica-Wagner et al. 2018) on the ground. The available DES $g, r, i, z, Y$ images are supplemented with deeper $g, r$, and near-infrared $H$-band images from the Magellan Telescopes to enable systematic studies of galaxy environments of individual absorbers.

To obtain a representative map of the dominant cosmic baryon reservoir at $z=0.4-1$, the targeted sample size is defined such that (1) a statistically representative sample of $\approx 100$ of $0.1 L_{*}-L_{*}$ galaxies at $z>0.4$ can be established for a comprehensive study of the CGM and (2) a large redshift pathlength is reached for IGM metal-absorption line surveys. Based on the best-fit luminosity functions for red and blue galaxies from Cool et al. (2012), we estimate that 15 QSO fields are needed for establishing a sample of $\approx 75$ blue, star-forming and $\approx 30 \mathrm{red}$, evolved galaxies over a wide range of luminosity at redshifts between $z_{\text {gal }} \approx 0.4$ and $z_{\text {gal }} \approx 0.8$ and projected distances $d<300$ pkpc from the QSO sightlines. In addition, a complete galaxy survey carried out in these CUBS fields will also double the number of $z<0.4$ galaxies with known CGM constraints. Furthermore, combining 15 new CUBS QSOs and available archival sightlines is expected to lead to the largest redshift survey pathlength of $\Delta z \approx 8$ (13) for high-ionization species probed by the $\mathrm{O}_{\text {vi }} \lambda \lambda 1031,1037$ and Ne viII $\lambda \lambda 770,780$ doublets at $z>0.4$. These represent new samples of $\mathrm{O}$ VI and NeviII absorbers that are statistically significant in size for robust measurements of the frequency distribution function and the cosmic mass density of these highly-ionized species. Because

1 https://www.darkenergysurvey.org/ 

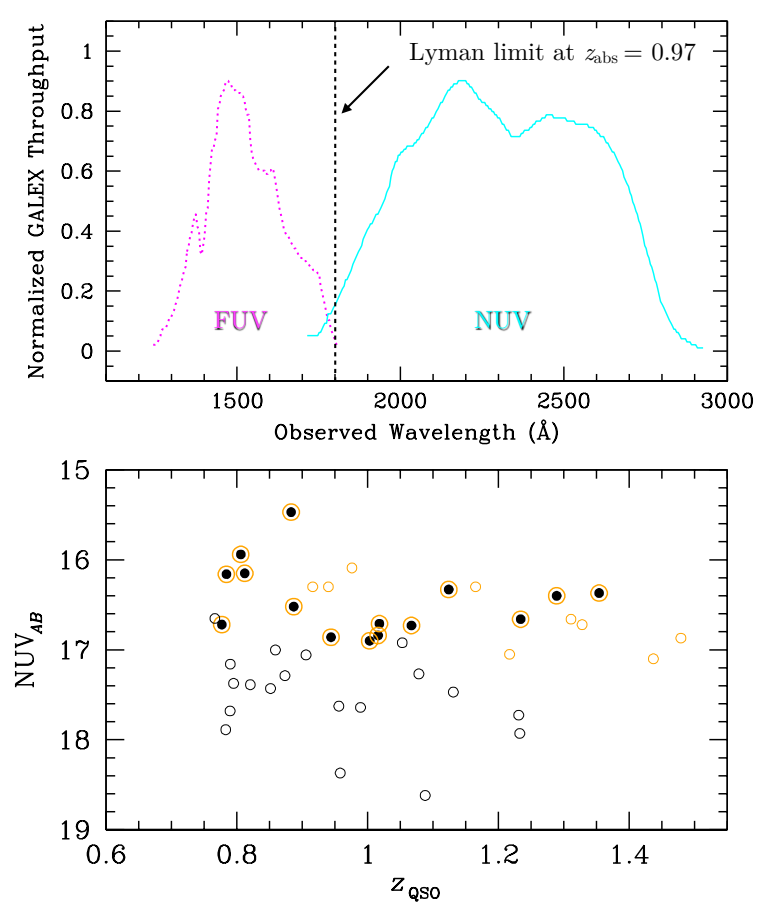

Figure 1. Top: Normalized throughput functions for the GALEX FUV (dotted magenta curve) and NUV (solid cyan curve) bandpasses. LLSs at $z_{\text {abs }} \lesssim 0.97$ attenuate the observed FUV flux in the background QSO. A FUV-bright QSO sample would therefore be biased against these low-redshift LLSs. Therefore, CUBS QSOs are selected based on their NUV brightness. Bottom: NUV magnitude versus redshift distribution of $z_{\mathrm{QSO}}>0.7$ QSOs with UV spectra available in the $H S T$ archive. Those with available highquality COS UV spectra of $\mathrm{S} / \mathrm{N} \gtrsim 15 \mathrm{resel}^{-1}$ are highlighted in orange circles, and those obtained as part of the CUBS program are shown in solid points.

the absorber samples are drawn from random sightlines, they probe diverse gaseous environments (i.e., from interstellar to circumgalactic and intergalactic space).

The QSO fields are selected blindly without prior knowledge of the line-of-sight galactic environment. A lesson learned from previous CGM experiments is that preferentially selecting QSO fields with a larger number of known photometrically-selected galaxies does not necessarily help increase the galaxy sample size for a fixed number of QSO fields but likely biases the galaxy sample toward galaxy groups (see for example Werk et al. 2012; Qu \& Bregman 2018). The inclusion of galaxy groups not only skews the galaxy sample toward more massive halos but also introduces ambiguities in interpreting the physical connections between the absorber and multiple group members. The QSOs in the CUBS program are presented in Table 1. Figure 1 (bottom panel) presents the NUV magnitude versus redshift distribution of known UV bright QSOs at $z_{\mathrm{QSO}}>0.7$. The CUBS QSOs are highlighted in solid points, showing a three-fold increase in the number of high-quality UV absorption spectra at $z_{\mathrm{QSO}}>0.7$.

\subsection{COS UV Spectroscopy}

Medium-resolution, high signal-to-noise ratio $(S / N)$ FUV spectra of 15 new NUV bright QSOs were obtained
Table 2. Journal of CUBS HST COS Observations

\begin{tabular}{|c|c|c|c|c|}
\hline \multirow[b]{2}{*}{ QSO } & \multirow[b]{2}{*}{$z_{\mathrm{QSO}}$} & \multicolumn{2}{|c|}{$t_{\exp }(\mathrm{sec})$} & \multirow[b]{2}{*}{$\langle S / N\rangle_{\text {resel }}$} \\
\hline & & $\mathrm{G}_{130 \mathrm{M}^{a}}$ & $\mathrm{G}_{160 \mathrm{M}^{b}}$ & \\
\hline J0028-3305 & 0.887 & 13253 & 17589 & 23 \\
\hline J0110-1648 & 0.777 & 15320 & 19802 & 31 \\
\hline J0111-0316 & 1.234 & 15149 & 19711 & 20 \\
\hline J0114-4129 & 1.018 & 15320 & 19798 & 12 \\
\hline J0119-2010 & 0.812 & 7049 & 13437 & 24 \\
\hline J0154-0712 & 1.289 & 9716 & 14843 & 28 \\
\hline J0248-4048 & 0.883 & 4516 & 5538 & 20 \\
\hline J0333-4102 & 1.124 & 12534 & 14849 & 24 \\
\hline J0357-4812 & 1.016 & 32664 & 24630 & 27 \\
\hline J0420-5650 & 0.944 & 17797 & 20570 & 22 \\
\hline J0454-6116 & 0.784 & 8330 & 13503 & 22 \\
\hline J2135-5316 & 0.806 & 7098 & 9738 & 18 \\
\hline $\mathrm{J} 2245-4931$ & 1.003 & 9395 & 19321 & 14 \\
\hline $\mathrm{J} 2308-5258$ & 1.067 & 20618 & 22118 & 23 \\
\hline J2339-5523 & 1.354 & 9388 & 14987 & 22 \\
\hline
\end{tabular}

under the CUBS program (PID $=15163$; PI: Chen) using COS on board the HST. COS with the G130M and G160M gratings and a combination of multiple central wavelength settings (see Table 2) offers a contiguous spectral coverage of $\lambda=1100-1800 \AA$, with a spectral resolution of Full-Width-at-Half-Maximum $\delta v_{\text {FWHM }} \approx 20 \mathrm{~km} \mathrm{~s}^{-1}$ for observing a wide range of ionic transitions at $z<1$. These include the hydrogen Lyman-series transitions from Ly $\alpha$ and $\operatorname{Ly} \beta$ onward to the Lyman-limit transition, and heavyelement transitions such as $\mathrm{O}_{\text {III }} \lambda 702$, Ne viI $\lambda \lambda 770,780$,

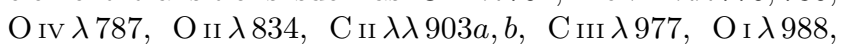
$\mathrm{O}_{\text {VI }} \lambda \lambda 1031,1037$, etc. The full coverage of the H I Lyman series enables precise and accurate measurements of the neutral hydrogen column density $N(\mathrm{HI})$. The relative abundances between different ions enable accurate estimates of the ionization state and metallicity of the gas.

All COS target acquisitions (TA) were performed using $S / N>50$ ACQ/IMAGEs. Analysis of the primary and confirmation images reveal that all targets were centered to better than $0.015^{\prime \prime}\left(0.016^{\prime \prime}\right)$ along the dispersion (cross-dispersion) direction. For G130M and G160M spectra, the dispersion velocity offsets due to TA are less than 1.5 $\mathrm{km} \mathrm{s}^{-1}$. A summary of the COS observations is presented in Table 2, which lists for each QSO, the name and redshift of the QSO, the total exposure time per grating in seconds, and the mean $S / N$ per resolution element, $\langle S / N\rangle_{\text {resel }}$ in the final combined spectrum.

Raw data from COS were reduced following standard pipeline procedures using $C A L C O S$ (v3.3.4 or v3.3.5). In addition, individual one-dimensional spectra were further processed and combined using custom software to ensure the accuracy of wavelength calibration and to optimize the $S / N$ in the final combined spectra. A detailed description of the software can be found in Chen et al. (2018). In summary, relative wavelength offsets between individual spectra were first determined using a low-order polynomial that best describes the offsets between common absorption lines found in different exposures. These wavelength-corrected spectra were then coadded to form a final combined spectrum using individual exposure times as weights. The final absolute wavelength calibration was guided by either the line- 
Table 3. Journal of CUBS Magellan MIKE Observations

\begin{tabular}{ccrrrrr}
\hline \hline & & \multirow{2}{*}{$V$} & \multirow{2}{*}{$t_{\exp }$} & \multirow{2}{*}{ FWHM } & \multicolumn{2}{c}{$\langle S / N\rangle_{\text {resel }}$} \\
\cline { 6 - 7 } QSO & & & & & & \\
& & & & & & \\
QSO & $(\mathrm{mag})$ & $(\mathrm{sec})$ & $(\mathrm{km} / \mathrm{s})$ & $3500 \AA$ & $4500 \AA$ \\
\hline $\mathrm{J} 0028-3305$ & 0.887 & 16.4 & 2100 & 8 & 6 & 35 \\
$\mathrm{~J} 0110-1648$ & 0.777 & 16.0 & 1800 & 8 & 12 & 38 \\
$\mathrm{~J} 0111-0316$ & 1.234 & 15.5 & 1800 & 8 & 18 & 55 \\
$\mathrm{~J} 0114-4129$ & 1.018 & 16.7 & 3600 & 8 & 10 & 42 \\
$\mathrm{~J} 0119-2010$ & 0.812 & 15.8 & 1800 & 8 & 16 & 55 \\
$\mathrm{~J} 0154-0712$ & 1.289 & 15.8 & 1500 & 8 & 14 & 42 \\
$\mathrm{~J} 0248-4048$ & 0.883 & 15.1 & 900 & 8 & 13 & 48 \\
$\mathrm{~J} 0333-4102$ & 1.124 & 15.8 & 2700 & 10 & 20 & 65 \\
$\mathrm{~J} 0357-4812$ & 1.016 & 16.0 & 5400 & 10 & 12 & 41 \\
$\mathrm{~J} 0420-5650$ & 0.944 & 16.1 & 3000 & 10 & 8 & 30 \\
$\mathrm{~J} 0454-6116$ & 0.784 & 15.8 & 1800 & 10 & 17 & 58 \\
$\mathrm{~J} 2135-5316$ & 0.806 & 15.8 & 3200 & 8 & 14 & 64 \\
$\mathrm{~J} 2245-4931$ & 1.003 & 16.5 & 3600 & 8 & 6 & 35 \\
$\mathrm{~J} 2308-5258$ & 1.067 & 16.2 & 5800 & 8 & 11 & 52 \\
$\mathrm{~J} 2339-5523$ & 1.354 & 15.5 & 2700 & 8 & 15 & 78 \\
\hline
\end{tabular}

of-sight velocity offset of the Milky Way Ca II H\&K lines or the redshift of a strong intervening $\mathrm{Mg}$ II absorber detected in the ground-based MIKE optical echelle spectrum of the QSO (see $\S 2.3$ below), which sets the wavelengths for the associated FUV transitions in the COS spectra. A mean wavelength zero point offset was then determined by registering the associated low-ionization lines observed in the COS spectra to the expected wavelength in vacuum. The final wavelength solution generated from our custom software was found to be accurate to within $\pm 5 \mathrm{~km} \mathrm{~s}^{-1}$, based on comparisons of the velocity centroids between low-ionization lines observed in COS spectra and those of $\mathrm{Mg}$ II $\lambda \lambda 2796,2803$ lines observed in higher-resolution ground-based echelle spectra $\left(\delta v_{\mathrm{FWHM}} \approx 8-10 \mathrm{~km} \mathrm{~s}^{-1}\right)$. Finally, each combined spectrum was continuum-normalized using a low-order polynomial fit to spectral regions free of strong absorption features. The final continuum-normalized spectra have a median $\langle S / N\rangle_{\text {resel }} \approx 12-31$ (see Table 2 ). The large variation in the $S / N$ of the final combined spectra is largely due to QSO variability. For example, comparing $S / N$ in the final COS spectra and known GALEX magnitudes of the QSOs, we estimate that J0110-1648 had brightened by a factor of $\approx 1.4$, while J0114-4129 had faded by a factor of 2 since the GALEX observations. Large QSO variability was also directly observed in the COS spectra of J2308-5258 obtained several months apart.

\subsection{Optical Echelle Spectroscopy}

We complement the FUV spectra from COS with optical echelle spectra of the QSOs, obtained using MIKE (Bernstein et al. 2003) on the Magellan Clay telescope. MIKE delivers an unbinned pixel resolution of $0.12^{\prime \prime}\left(0.13^{\prime \prime}\right)$ along the spatial direction and $\approx 0.02(0.05) \AA$ along the spectral direction in the blue (red) arm, covering a wavelength range of $\lambda=3200-5000$ (4900-9200) $\AA$. It provides extended spectral coverage for additional ionic transitions through observations of the Fe II absorption series, the $\mathrm{Mg}$ II $\lambda \lambda 2796,2803$ doublet features, $\mathrm{Mg}$ I $\lambda 2852$, and Ca II $\lambda \lambda$ 3934, 3969 absorption, and enables accurate relative abundances studies (e.g., Zahedy et al. 2016, 2017).

The majority of the optical echelle spectra of the CUBS QSOs were obtained between September 2017 and March 2018, with additional observations taken in February 2019
Table 4. Journal of Completed MUSE-LLS Observations

\begin{tabular}{lcccc}
\hline \hline QSO & $\begin{array}{c}t_{\exp } \\
(\mathrm{sec})\end{array}$ & $\begin{array}{c}\text { FWHM } \\
(\operatorname{arcsec})\end{array}$ & $\begin{array}{c}A B(r)^{a} \\
(\mathrm{mag})\end{array}$ & $\begin{array}{c}\mathrm{SB}(7000 \AA)^{b} \\
\mathrm{erg} / \mathrm{s} / \mathrm{cm}^{2} / \AA / \mathrm{arcsec}^{2}\end{array}$ \\
\hline $\mathrm{J} 0248-4048^{c}$ & 7650 & 0.7 & 27.0 & $9.8 \times 10^{-20}$ \\
$\mathrm{~J} 0357-4812$ & 9390 & 0.6 & 27.4 & $7.5 \times 10^{-20}$ \\
$\mathrm{~J} 2135-5316$ & 6840 & 0.6 & 26.9 & $1.3 \times 10^{-19}$ \\
\hline${ }^{\mathrm{a}} 5-\sigma$ limiting magnitude in the pseudo $r$-band integrated from $6000 \AA$ \\
to $7000 \AA$. \\
b $1-\sigma$ limiting surface brightness at 7000 $\AA$ per sq. arcsecond aperture \\
c One of the OBs for J0248-4048 was obtained through clouds. While \\
the exposures through clouds do not reach the same depth as those \\
obtained under clear skies, including all exposures yields the deepest \\
combined data cube for this field.
\end{tabular}

and October 2019. These UV-bright QSOs are also bright in the optical window, with $V$-band magnitude ranging from $V=15.1 \mathrm{mag}$ to $V=16.5 \mathrm{mag}$. The echelle spectroscopy of CUBS QSOs was carried out as a filler program within other regular programs. As a result, two readout settings, $2 \times 2$ versus $3 \times 3$ binning, were adopted for the QSO sample, leading to a spectral resolution of $\delta v_{\mathrm{FWHM}} \approx 8 \mathrm{~km} \mathrm{~s}^{-1}$, and $10 \mathrm{~km} \mathrm{~s}^{-1}$, respectively. The echelle spectra were processed and extracted using custom software described in Chen et al. (2014) and in Zahedy et al. (2016). Wavelength calibrations were performed using a ThAr frame obtained immediately after each science exposure and subsequently corrected to a vacuum and heliocentric wavelength scale. Relative flux calibrations were performed using a sensitivity function determined from a spectrophotometric standard star observed on the same night as the CUBS QSOs. Individual flux-calibrated echelle orders from different exposures were then coadded and combined to form a single final spectrum. Finally, the combined spectrum was continuum-normalized using a low-order polynomial fit to the spectral regions free of strong absorption features.

A summary of available optical echelle spectra is presented in Table 3, which lists for each QSO the $V$-band magnitude, the total accumulated exposure time, spectral resolution, and the mean $S / N$ per resolution element at $\lambda=3500$ and $4500 \AA$. The mean $S / N_{\text {resel }}$ of the final combined spectra ranges between 6 and 20 at $\lambda=3500 \AA$ and between 30 and $\approx 80$ at $\lambda=4500 \AA$. These echelle spectra offer a factor of two larger resolving power for metal lines than the FUV spectra from COS. They serve as an important guide for analyzing the COS spectra.

\subsection{MUSE Observations}

The ultradeep galaxy survey component described at the beginning of $\S 2$ is being carried out using the MultiUnit Spectroscopic Explorer (MUSE; Bacon et al. 2010) on the VLT UT4 in service mode under program ID, 0104.A0147 (PI: Chen). MUSE observes a field of $1^{\prime} \times 1^{\prime}$ with a plate scale of $0.2^{\prime \prime}$ and $1.25 \AA$ per pixel, covering a spectral range from $4800 \AA$ to $9200 \AA$ with a spectral resolution of $\delta v_{\mathrm{FWHM}} \approx 120 \mathrm{~km} \mathrm{~s}^{-1}$ at $7000 \AA$. The combined spatial and spectral resolving power of MUSE provides high sensitivity and high efficiency for surveys of distant faint galaxies and line-emitting nebulae, and is uniquely suitable for uncovering faint emission close to QSO sightlines (e.g., Schroetter et al. 2016; Bielby et al. 2017; Johnson et al. 2018; Péroux et al. 2019; Chen et al. 2019a,b). For the CUBS QSO fields, 

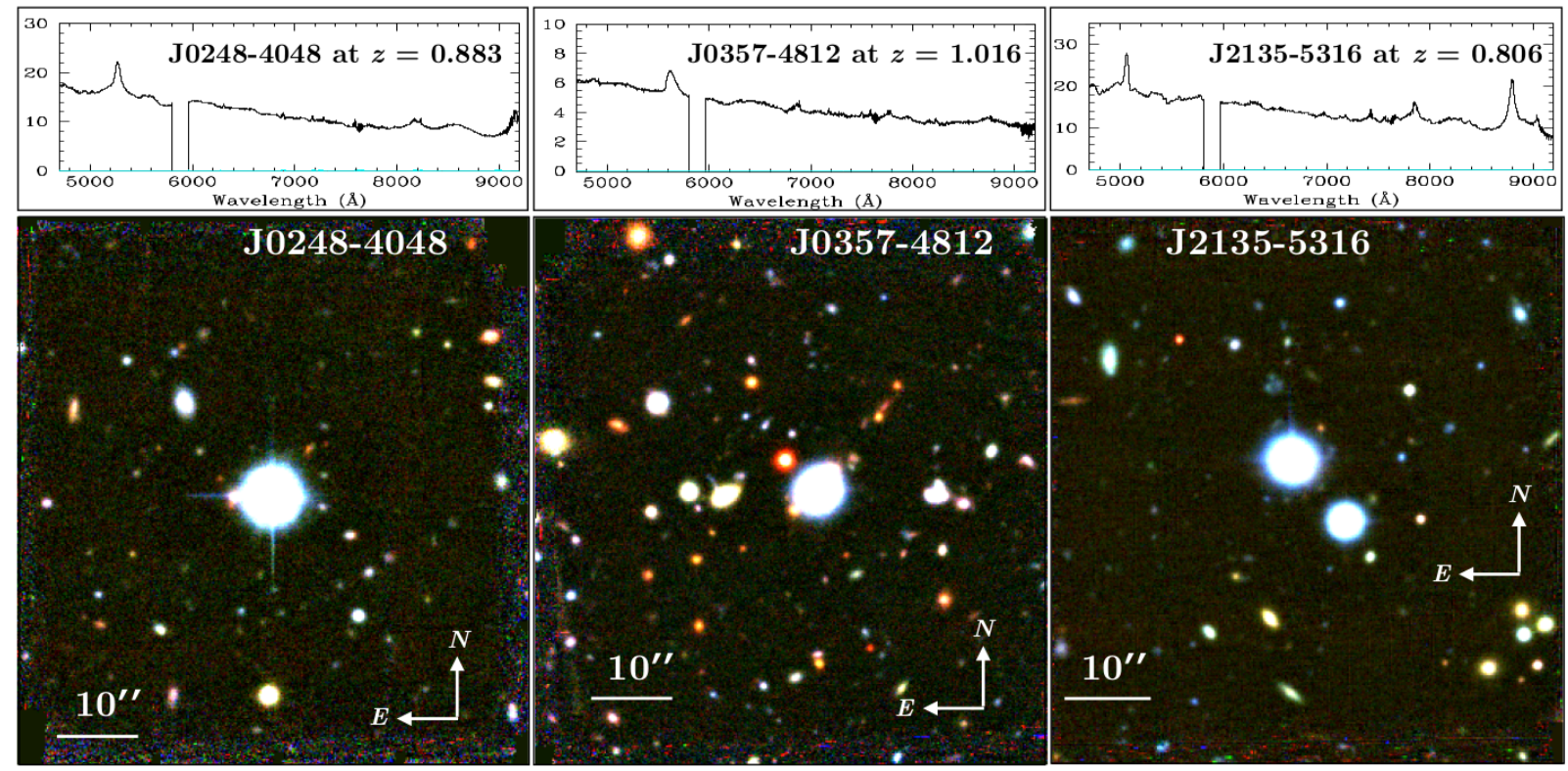

Figure 2. MUSE observations of three CUBS QSO fields. Optical spectra of the QSOs extracted from the combined MUSE data cube are presented at the top, showing the spectral coverage of MUSE. The spectral gap at 5800-5965 $\AA$ in the top panels is due to the sodium filter, which was applied to block the scattered light from the laser beams for wavefront corrections. Continuum images of the QSO fields are presented at the bottom. The color images are produced from integrating the MUSE cube over wavelength ranges of $4800-5800 \AA$ (pseudo $g$-band), 6000-7000 $\AA$ (pseudo $r$-band), and 7500-8500 $\AA$ (pseudo $i$-band). For each field, north is up and east is to the left. The QSO is at the center, and the horizontal bar in the lower-left corner marks $10^{\prime \prime}$ on the sky.

the combination of $H S T$ COS absorption spectroscopy and MUSE integral-field spectroscopy provides a powerful tool for studying the complex interplay between gas and galaxies at $z \lesssim 1$.

The CUBS-MUSE program aims to uncover galaxies as faint as $\approx 0.01 L_{*}$ at $z \lesssim 1$ near the QSO sightlines. The observations are carried out in wide-field mode (WFM) with adaptive optics assistance. While the use of lasers for wavefront corrections imposes a gap in the spectral coverage from 5800 to $5965 \AA$, it ensures a uniform mean image quality of FWHM $<0.8^{\prime \prime}$ across all fields. For each CUBS QSO field, the MUSE observations are carried out in a series of two to three observing blocks (OBs) with individual OBs consisting of three exposures of 850 to $1130 \mathrm{~s}$ each. To optimize cosmic-ray rejection and minimize a fixed residual flat-field pattern at the edges of individual slicers, a small dither of $\approx 2^{\prime \prime}-4^{\prime \prime}$ and a relative field rotation of $90^{\circ}$ are applied to every successive exposure. While the program is ongoing, here we present results from three completed CUBS QSO fields, all with a LLS found in the foreground (see $\S 3$ below). The QSO fields and the total accumulated exposure time with MUSE are presented in the first two columns of Table 4.

All MUSE data cubes are reduced using a combination of the standard ESO MUSE pipeline (Weilbacher et al. 2014) and CUBEXTRACTOR, a custom package developed by S. Cantalupo (see Cantalupo et al. 2019 for a detailed description). For each OB, both raw science exposures and the associated raw calibration files, including bias, flats, comparison arc files, and spectrophotometric standard, are retrieved from the ESO science archive. The ESO MUSE pipeline first generates a master bias and a master flat, processes the arcs for wavelength calibration and for measuring the instrument line spread function, and twilight flats for il- lumination corrections. Then it applies these calibrations to the standard star and science frames to produce a response function, telluric corrections, and a sky continuum using the $20 \%$ darkest pixels in the field of view. Finally, the pipeline produces a sky-subtracted 3D data cube for each raw science exposure, along with associated pixel tables storing all calibration parameters, and a whitelight image for object identifications. Individual data cubes are then registered using common objects identified in the corresponding whitelight image to form a final combined cube for each OB.

The final product from the standard ESO pipeline exhibits apparent residual patterns in the sky background due to imperfect illumination correction and sky subtraction (see Figure 1 of Lofthouse et al. 2020 for an example). The residuals are most severe near/at bright sky lines, making robust identifications of faint features challenging. In particular, MUSE consists of 24 integral field units (IFUs) and each IFU consists of four stacks of 12 slices. Any temporal and wavelength-dependent changes in the illumination pattern and instrument line spread function result in large background residuals in the final data cube. To improve upon the standard pipeline reduction, the CUBEXTRACTOR package first adopts the pipeline produced data cube with sky subtraction turned off to define a reference grid for the postprocessing steps and resamples the pixel tables on to the reference grid. Then "CubeFix" works in the reconstructed data cube space and empirically determines a wavelengthdependent illumination pattern per image slice using the sky background observed in each science frame. Next, "CubeSharp" performs a flux-conserving (across the field of view) sky subtraction routine using an empirical line spread function constructed from the skylines recorded in the science frame. These steps are performed twice using an updated 
object catalog for masking continuum sources in the second iteration of sky removal. Finally, individually corrected data cubes from all OBs are combined to form a final data cube. Because the MUSE wavelength solution is calibrated in air and QSO absorption spectra are calibrated to vacuum, the product of CUBEXTRACTOR is further resampled to vacuum wavelength to facilitate an accurate redshift comparison between galaxies identified in MUSE and absorbers identified in the COS spectra.

The QSO spectra and RGB images constructed using pseudo $g_{-}, r_{-}$, and $i$-band images integrated over wavelength windows of 4800-5800 $⿱$, 6000-7000 , and 7500-8500 , respectively, from the final MUSE data cubes of the three CUBS QSO fields are presented in Figure 2. The image quality in the final combined cubes ranges between FWHM $\approx$ $0.6^{\prime \prime}$ and $0.7^{\prime \prime}$ at $7000 \AA$. The image quality is slightly worse at shorter wavelengths, ranging from $\approx 0.7^{\prime \prime}$ to $0.8^{\prime \prime}$ at 5000 $\AA$, and better at longer wavelengths, ranging from $\approx 0.56^{\prime \prime}$ to $0.6^{\prime \prime}$ at $9000 \AA$. The 5 - $\sigma$ limiting magnitude in the pseudo $r$-band ranges between $r=27.0$ and 27.4 mag over $1^{\prime \prime}$ diameter aperture and the 1- $\sigma$ limiting surface brightness reaches $(0.8-1.3) \times 10^{-19} \mathrm{erg} \mathrm{s}^{-1} \mathrm{~cm}^{-2} \AA^{-1} \operatorname{arcsec}^{-2}$ at $7000 \AA$ per square arcsecond aperture (see Table 4).

\section{SURVEY OF LYMAN LIMIT ABSORBERS AT $Z<1$}

The CUBS QSOs are selected based on the QSO emission redshift with $z_{\mathrm{QSO}} \gtrsim 0.8$ and the NUV brightness with $\mathrm{NUV}_{\approx}<16.9$ (Figure 1 ), with no prior knowledge of the line-ofsight absorption properties or galactic environment. Therefore, the CUBS QSO sample provides a uniform sample (cf. archival samples from Ribaudo et al. 2011 or Shull et al. 2017) for studying the incidence of optically-thick gas at $z \lesssim 1$ as well as the connection between LLS and galaxy properties. The COS spectra described in $\S 2.2$ are of sufficiently high quality with $S / N_{\text {resel }}>12$ to enable a robust identification of both the Lyman discontinuity at $\approx 912 \AA$ and the Lyman series lines at longer wavelengths. Here we describe the procedures we use to identify these absorbers and to measure $N\left(\mathrm{H}_{\mathrm{I}}\right)$.

\subsection{The search for Lyman continuum breaks}

The search for LLS in the CUBS QSO sample is carried out using the following steps. For each QSO sightline, a minimum redshift $z_{\text {min }}$ for the LLS search is defined by the minimum wavelength where $S / N_{\text {resel }}>3$, while the maximum survey redshift $z_{\max }$ is defined by either the maximum wavelength of the COS spectrum or the emission redshift of the QSO, excluding the velocity window of $|\Delta v|=3000$ $\mathrm{km} \mathrm{s}^{-1}$ from $z_{\mathrm{QSO}}$ to avoid the QSO proximity zone where the ionizing radiation intensity is expected to be enhanced due to the background QSO (e.g., Pascarelle et al. 2001; Wild et al. 2008). Table 5 summarizes $z_{\min }$ and $z_{\max }$ for each CUBS QSO, along with the redshift survey pathlength $\Delta z_{\mathrm{LL}}$, in columns (2)-(4). Together, the 15 CUBS QSOs provide a total redshift survey pathlength of $\Delta z_{L L}=9.3$ for new LLSs.

Each Lyman limit absorber is then identified based on an apparent flux discontinuity in the QSO spectrum and
Table 5. Summary of new (p)LLSs in the CUBS fields

\begin{tabular}{cccccr}
\hline \hline $\begin{array}{c}\text { Field } \\
(1)\end{array}$ & $\begin{array}{c}z_{\min } \\
(2)\end{array}$ & $\begin{array}{c}z_{\max } \\
(3)\end{array}$ & $\begin{array}{c}\Delta z_{\mathrm{LL}} \\
(4)\end{array}$ & $\begin{array}{c}z_{\mathrm{abs}} \\
(5)\end{array}$ & \multicolumn{1}{c}{$\begin{array}{c}\tau_{912} \\
(6)\end{array}$} \\
\hline $\mathrm{J} 0028-3305$ & 0.21 & 0.87 & 0.66 & $\ldots$ & $\ldots$ \\
$\mathrm{J} 0110-1648$ & 0.20 & 0.76 & 0.56 & $0.4723^{a}$ & $0.18 \pm 0.01$ \\
& & & & $0.5413^{a}$ & $0.21 \pm 0.01$ \\
$\mathrm{~J} 0111-0316$ & 0.57 & 0.94 & 0.37 & $0.5762^{b}$ & $>6.61$ \\
$\mathrm{~J} 0114-4129$ & 0.23 & 0.94 & 0.71 & 0.3677 & $0.16 \pm 0.02$ \\
& & & & 0.9001 & $0.41 \pm 0.03$ \\
$\mathrm{~J} 0119-2010$ & 0.21 & 0.79 & 0.58 & $\ldots$ & $\ldots$ \\
$\mathrm{J} 0154-0712$ & 0.20 & 0.94 & 0.74 & 0.3743 & $0.24 \pm 0.01$ \\
$\mathrm{~J} 0248-4048$ & 0.24 & 0.86 & 0.62 & 0.3640 & $2.48 \pm 0.01$ \\
$\mathrm{~J} 0333-4102$ & 0.20 & 0.94 & 0.74 & 0.9372 & $0.61 \pm 0.02$ \\
$\mathrm{~J} 0357-4812$ & 0.21 & 0.94 & 0.73 & 0.4353 & $0.99 \pm 0.01$ \\
$\mathrm{~J} 0420-5650$ & 0.21 & 0.92 & 0.71 & $\ldots$ & $\ldots$ \\
$\mathrm{J} 0454-6116$ & 0.21 & 0.76 & 0.55 & $\ldots$ & $\ldots$ \\
$\mathrm{J} 2135-5316$ & 0.62 & 0.79 & 0.17 & 0.6226 & $>6.27$ \\
$\mathrm{~J} 2245-4931$ & 0.22 & 0.94 & 0.72 & $\ldots$ & $\ldots$ \\
$\mathrm{J} 2308-5258$ & 0.22 & 0.94 & 0.72 & 0.2603 & $0.58 \pm 0.02$ \\
& & & & 0.5427 & $2.53 \pm 0.01$ \\
$\mathrm{~J} 2339-5523$ & 0.21 & 0.94 & 0.73 & $\ldots$ & $\ldots$ \\
\hline
\end{tabular}

${ }^{a}$ see Cooper et al. (2020)

$b_{\text {see Boettcher et al. (2020) }}$

verified based on the presence of associated Lyman series lines. A total of 12 such absorbers are found along nine of the 15 QSO sightlines, with the remaining six sightlines displaying no evident continuum breaks in the COS spectral window ${ }^{2}$. A mean opacity, $\tau_{912}$, is determined based on the observed flux decrement at $911.76 \AA$ relative to the expected continuum flux from extrapolating a linear model that best describes the continuum at rest-frame 920-923 $\AA$ at the redshift of the absorber, $z_{\text {abs }}$. The error in $\tau_{912}$ is estimated including uncertainties in both the continuum model and the measurement uncertainties in the mean flux observed at rest-frame $911.76 \AA$. The results, including $z_{\text {abs }}$, are presented in columns (5)-(6). Of the 12 absorbers identified based on an apparent Lyman discontinuity, five are LLSs with $\tau_{912} \gtrsim 1$ at $z_{\text {abs }}=0.36-0.62$ and five are partial LLSs (pLLSs) with $\tau_{912}$ from 0.2 to $\lesssim 1$ at $z_{\text {abs }}=0.26-0.94$. Figure 3 presents the full Lyman series spectra of the five new LLSs from this search in descending order of $N(\mathrm{HI})$.

\subsection{Measurements of $N\left(\mathrm{H}_{\mathrm{I}}\right)$ and $b_{\mathrm{HI}}$}

In addition to the prominent Lyman discontinuity, Figure 3 also shows that each of these new LLSs is resolved into multiple components of varying absorption strength. The resolved component structure is clearly displayed in the velocity profiles of both $\mathrm{H}_{\mathrm{I}}$ and the associated metal lines presented in Figure 4. In particular, higher-resolution $\left(\delta v_{\text {FWHM }} \approx 8 \mathrm{~km} \mathrm{~s}^{-1}\right.$ ) ground-based optical echelle spectra show that the associated Mg II doublets of these LLSs are resolved into between two and six well-defined components.

To obtain accurate measurements of $N(\mathrm{H}$ I $)$ for individual components, we perform a Voigt profile analysis that takes into account the full Lyman series lines and the observed flux discontinuity at the Lyman limit (see also Chen et al. 2018; Zahedy et al. 2019). We first generate a model

2 We note the presence of a likely LLS at $z_{\text {abs }} \approx 1$ toward J2339-5523 based on the observed spectral slope of the QSO and a suite of absorption transitions in the COS data. This system will be presented in Johnson et al. (in preparation). 


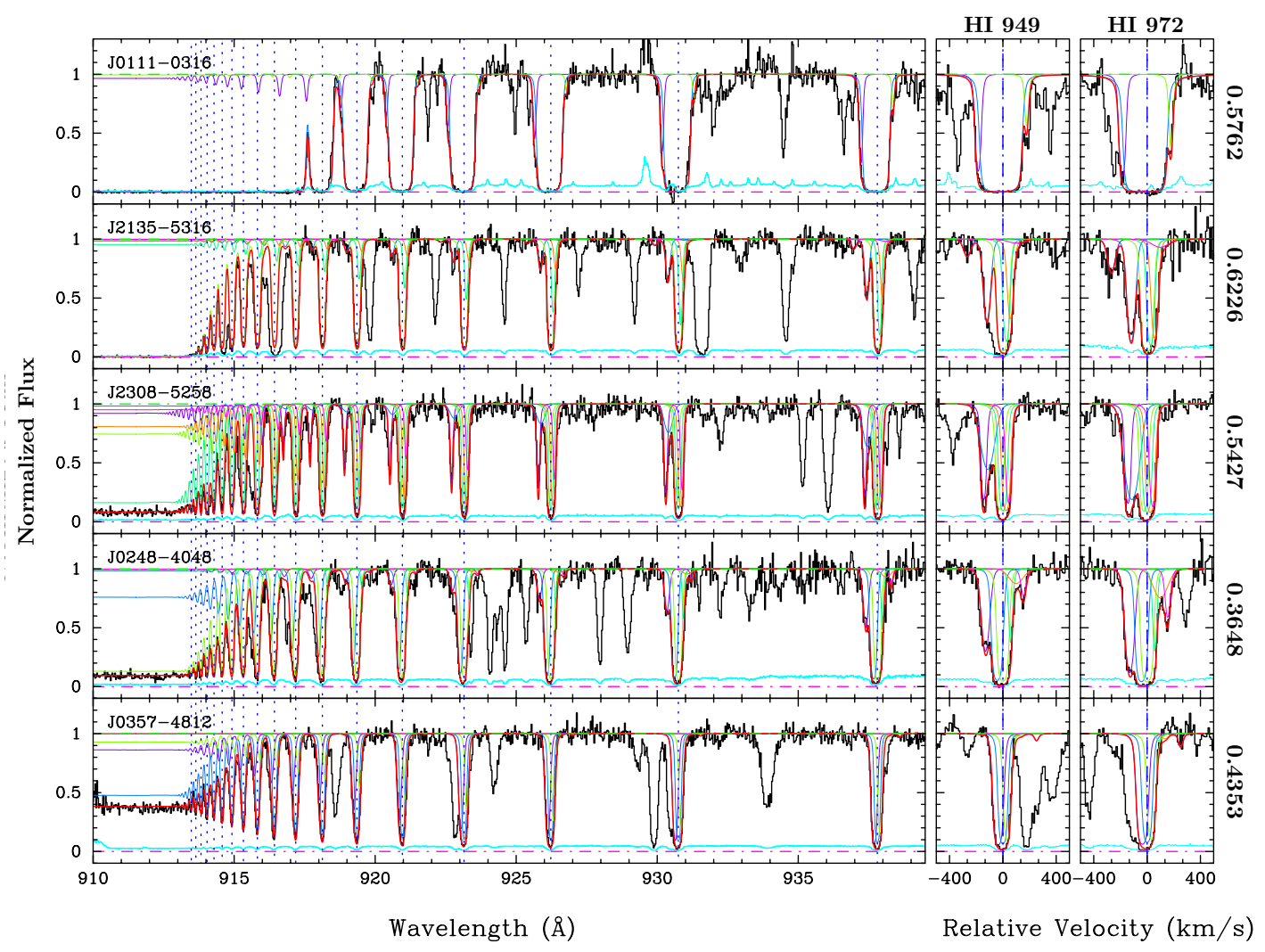

Figure 3. The Lyman series absorption spectra of the five new optically-thick H I absorbers with $\tau_{912} \gtrsim 1$ found in the CUBS QSO sightlines. The absorbers are ordered with decreasing log $N(\mathrm{H} \mathrm{I})$ from top to bottom. The continuum-normalized spectra are shown in black with the corresponding 1- $\sigma$ error shown in cyan. The green and magenta dash-dotted lines mark the normalized continuum and zero flux levels for guidance. For each absorber, the velocity profiles of Ly $\delta$ (H I 949) and Ly $\gamma$ (H I 972 ) are presented in the two right panels with zero velocity corresponding to the strongest H I component in Table 6. The remaining higher-order Lyman series lines, along with the Lyman limit, are presented in the left panel with the vertical blue dotted lines indicating the expected positions of the Lyman transitions. The best-fit Lyman series spectra are shown in red with individual components displayed in different colors.

absorption spectrum based on the minimum number of discrete components required to explain the observed absorption profiles. This process is guided by the component structure of the associated Mg II doublet for each LLS. Each component is characterized by three parameters: $N(\mathrm{HI}), b_{\mathrm{HI}}$, and the velocity centroid $d v_{c}$ relative to the redshift of the strongest $\mathrm{H}_{\mathrm{I}}$ absorbing component $z_{\text {abs }}$. The model Lyman series spectrum is then convolved with the COS line spread function (LSF) appropriate for Lifetime Position 4, at which the spectra were recorded. Next, we perform a $\chi^{2}$ minimization routine to determine the best-fit model parameters by comparing the LSF-convolved model spectrum with observations. For $\mathrm{H}$ I components with associated $\mathrm{Mg}$ II, $d v_{c}$ is fixed at the location determined from the centroid of the $\mathrm{Mg}$ II component. For $\mathrm{H}_{\text {I }}$ components without detected $\mathrm{Mg}$ II, $d v_{c}$ is allowed to vary during the $\chi^{2}$ minimization routine ${ }^{3}$.

3 One exception is the absorber at $z_{\text {abs }}=0.5762$ toward J0111-0316, which turns out to be an $\mathrm{H}_{2}$-bearing DLA, for which all available Lyman series lines are highly saturated, preventing us from resolving individual $\mathrm{H}_{\mathrm{I}}$ components within $\left|d v_{c}\right| \lesssim 150$ $\mathrm{km} \mathrm{s}^{-1}$, while the associated metal lines, such as the $\mathrm{Mg}$ II doublet, are resolved into seven discrete components in available optical echelle spectra (see Boettcher et al. 2020) for details.
To estimate the uncertainties associated with the bestfit parameters, we perform a Markov Chain Monte Carlo (MCMC) analysis using the EMCEE package (ForemanMackey et al. 2013). The MCMC analysis consists of 300 steps with an ensemble of 250 walkers, initialized over a small region in the parameter space around the minimum $\chi^{2}$ value. The first 100 steps of each walker are discarded when constructing a probability distribution function for each best-fit model parameter from combining results from all 250 walkers. The MCMC approach enables a robust evaluation of correlated errors between blended components over a reasonable amount of computing time. The results of the Voigt profile analysis are summarized in Table 6, where for each LLS the best-fit redshift of the strongest $\mathrm{H}$ i component $z_{\text {abs }}$ and the total $N\left(\mathrm{H}_{\mathrm{I}}\right)$ summed over all components are listed, along with the best-fit velocity centroid $d v_{c}$ relative to $z_{\text {abs }}, N_{c}\left(\mathrm{H}_{\mathrm{I}}\right), b_{c}\left(\mathrm{H}_{\mathrm{I}}\right)$ and associated 1- $\sigma$ uncertainties for individual components.

The best-fit Voigt profiles of individual $\mathrm{H}_{\mathrm{I}}$ components are shown in Figure 3 for the Lyman series from Ly $\gamma$ to the Lyman break, with the red spectrum representing the integrated profile over all components. The best-fit models are also displayed in the top two rows of Figure 4 for $\operatorname{Ly} \beta$ and $\operatorname{Ly} \gamma$ to contrast the velocity structures displayed in the associated ionic transitions. In all five LLSs, 


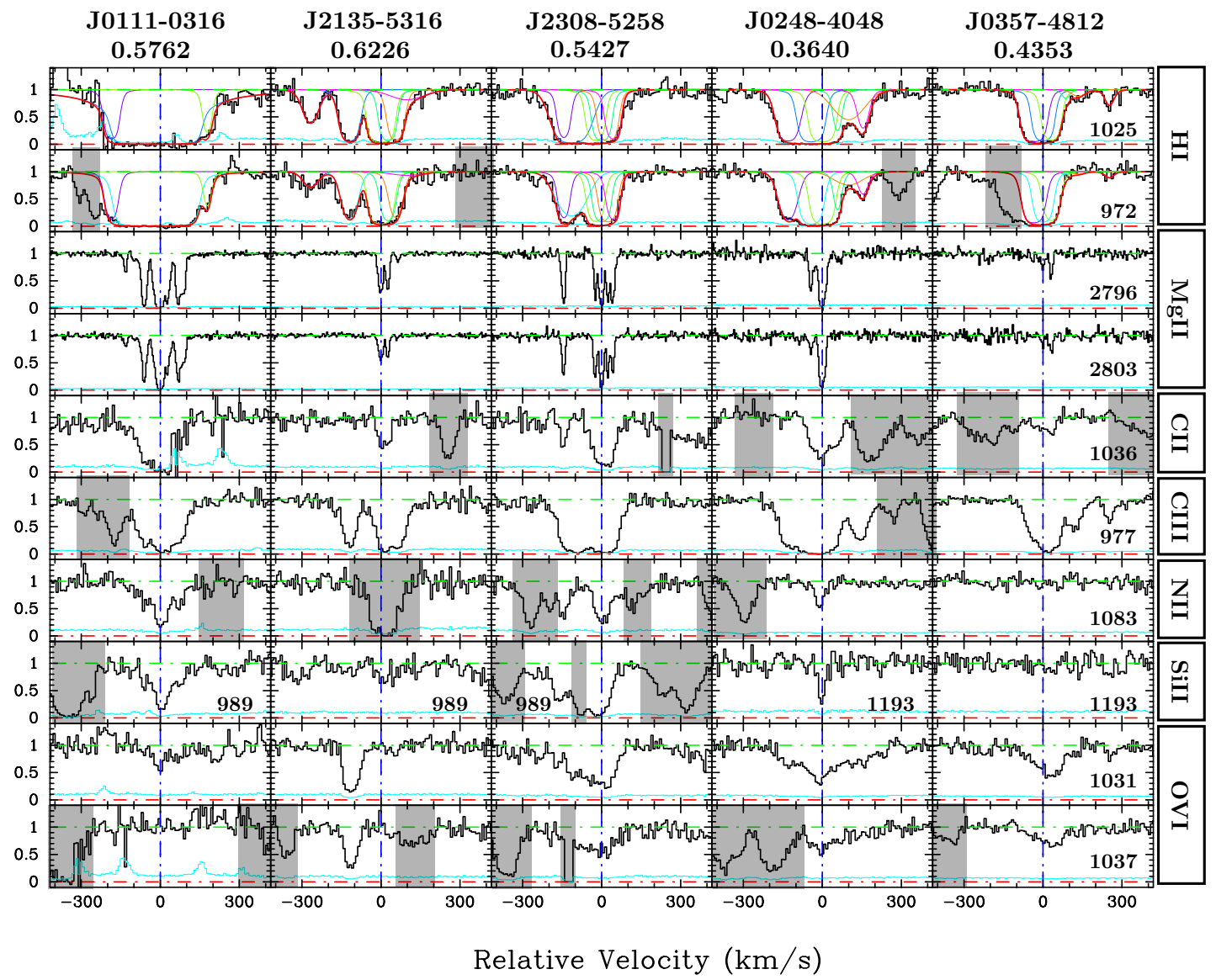

Figure 4. Absorption profiles of $\mathrm{Ly} \beta$ and $\mathrm{Ly} \gamma$, and associated Mg II, C II, C III, N II, Si II, and O VI found in the LLSs presented in Figure 3. The absorbers are ordered with decreasing $\log N(\mathrm{HI})$ from left to right. The rest-frame wavelength of each transition is listed in the lower-right corner of each panel in the right column. Following Figure 3, zero velocity corresponding to the redshift of the strongest $\mathrm{H}$ I component in Table 6, 1- $\sigma$ errors are shown in cyan, and spectral regions that are contaminated with blended absorption features are greyed out for clarity. In particular, the position where the N II $\lambda 1083$ line is expected for the LLS at $z_{\text {abs }}=0.6226$ toward J2135-5316 is dominated by a strong $\operatorname{Ly} \alpha$ absorber at $z_{\mathrm{abs}}=0.4468$. The best-fit Voigt profiles of $\mathrm{Ly} \beta$ and $\operatorname{Ly} \gamma$ lines, both separately for individual components (thin lines) and together for all components combined (thick red line), of each absorber are also reproduced in the two first rows for direct comparisons with resolved metal-line components.

a dominant component, containing between $75 \%$ and $98 \%$ of the total $\mathrm{H}$ i column density, is needed to explain the high-order Lyman series profiles, while additional satellite components are needed to explain the widths and asymmetric profiles observed in lower-order Lyman lines such as Ly $\alpha$ through Ly $\delta$. With the exception of the LLS at $z_{\text {abs }}=0.4353$ toward J0357-4812, the dominant H I component also corresponds to the strongest component observed in low-ionization transitions such as $\mathrm{C}$ II, N II, Mg II, and Si II. In particular, the associated Mg II components are fully resolved in the ground-based optical echelle spectra. To facilitate direct comparisons between $N\left(\mathrm{H}_{\mathrm{I}}\right)$ and ion abundances of different components, we also present initial measurements of $\mathrm{Mg}$ II component column densities, $N_{c}$ ( $\mathrm{Mg}$ II), in Table 6, but details regarding the Voigt profile analysis of metal absorption lines are presented in Zahedy et al. (2020, in preparation). The best-fit $b_{c}\left(\mathrm{HI}_{\mathrm{I}}\right)$ of the dominant $\mathrm{H}_{\text {I }}$ component ranges between $b_{c}\left(\mathrm{H}_{\mathrm{I}}\right) \approx 14 \mathrm{~km} \mathrm{~s}^{-1}$ and $b_{c}\left(\mathrm{H}_{\mathrm{I}}\right) \approx 20 \mathrm{~km} \mathrm{~s}^{-1}$, with the exception of the $\mathrm{H}_{2}$-bearing DLA at $z_{\text {abs }}=0.5762$ toward J0111-0316 for which the $\mathrm{H} \mathrm{I}$ components are fully blended (see Boettcher et al. 2020).
Because $b_{c}\left(\mathrm{HI}_{\mathrm{I}}\right) \equiv \sqrt{12.9^{2} T_{4}+b_{\text {turb }}^{2}} \mathrm{~km} \mathrm{~s}^{-1}$ for $\mathrm{H}_{\mathrm{I}}$ gas of temperature $T_{\text {gas }} \equiv 10^{4} \times T_{4}$ and turbulent width $b_{\text {turb }}$, the best-fit values constrain the underlying gas turbulence or bulk motion to be $b_{\text {turb }}<15 \mathrm{~km} \mathrm{~s}^{-1}$ for optically-thick absorbers of $T_{\text {gas }} \sim 10^{4} \mathrm{~K}$ and still smaller for warmer temperatures.

\section{DESCRIPTIONS OF INDIVIDUAL SYSTEMS}

The LLS survey described in $\S 3$ has yielded five new LLSs at $z_{\text {abs }}=0.3640-0.6226$, including one damped Ly $\alpha$ absorber (DLA) which also contains molecular hydrogen (see $\S 4.1$ below). A suite of ionic transitions is detected in these high $N\left(\mathrm{H}_{\mathrm{I}}\right)$ absorbers, including low-ionization transitions such as $\mathrm{C}_{\text {II, }} \mathrm{Mg}$ II, Si II, and Fe II, and intermediateionization transitions such as $\mathrm{C}_{\text {III }}$ and/or $\mathrm{N}$ III. With the exception of the $\mathrm{H}_{2}$-bearing DLA, the other four LLSs also exhibit strong associated Ovi doublets (Figure 4). The observed relative column density ratios between low- and intermediate-ionization transitions indicate that the gas is 


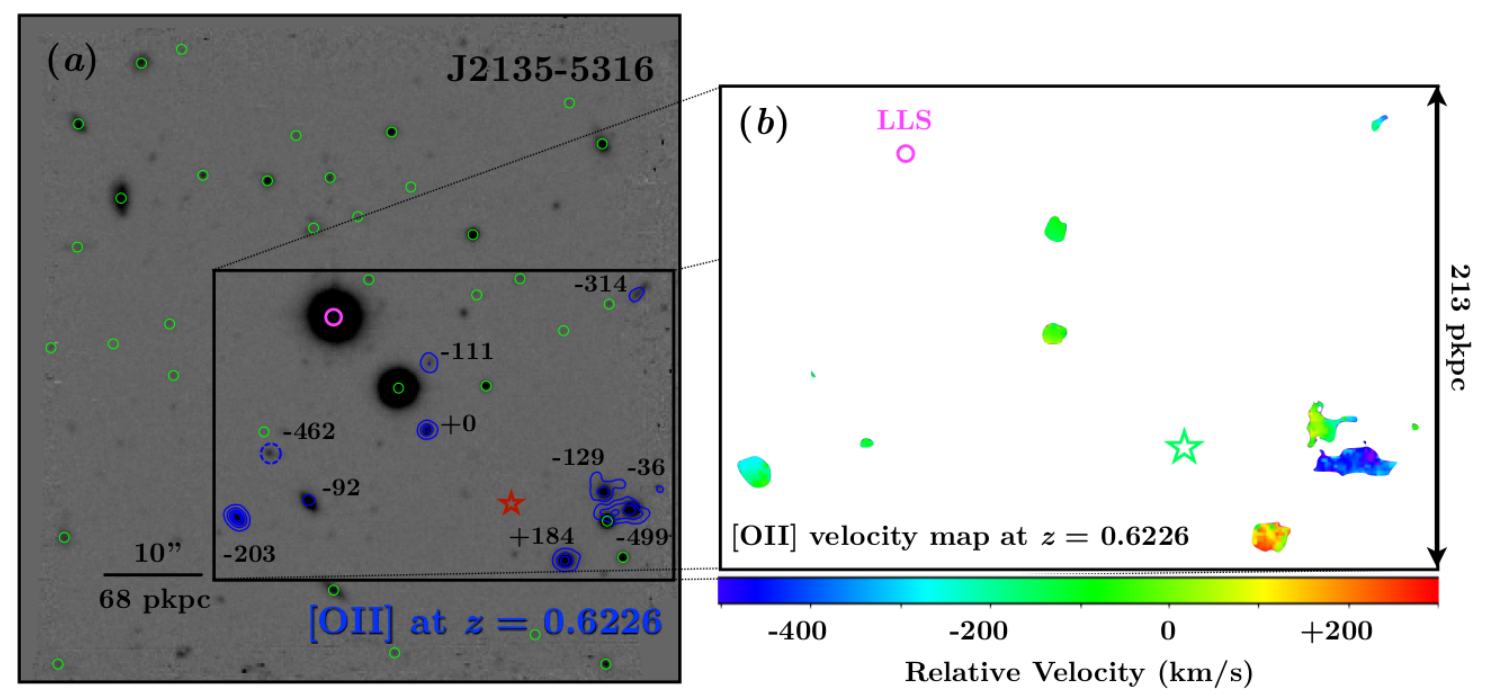

Figure 5. The galaxy environment uncovered by MUSE for the LLS at $z_{\text {abs }}=0.6226$ toward J2135-5316. The pseudo $r$-band image from Figure 2 is reproduced in panel $(a)$. North is up and east is to the left. Objects that are spectroscopically identified at cosmologically distinct redshifts from the LLS are marked by green circles, while galaxies spectroscopically identified in the vicinity of the LLS are highlighted by their blue [O II] emission contours of constant surface brightness $0.25,1.25,2.5,3.7$, and $5 \times 10^{-17} \mathrm{erg} \mathrm{s}^{-1} \mathrm{~cm}^{-2}$ arcsec ${ }^{-2}$ and marked by their line-of-sight velocity offset $\left(\mathrm{km} \mathrm{s}^{-1}\right)$ from $z_{\mathrm{abs}}=0.6226$. The galaxy at $\Delta v=-462 \mathrm{~km} \mathrm{~s}^{-1}$ exhibits only a faint trace of [O II] emission, with surface flux density below the lowest contour. It is marked by a blue, dashed circle. A group of 10 galaxies is found in the vicinity of the LLS with angular distance ranging from $\theta=10.6^{\prime \prime}$ to $38.1^{\prime \prime}$ from the QSO sightline (magenta circle), corresponding to a range in projected distance from $d=72$ to $252 \mathrm{pkpc}$ at the redshift of the LLS. The light-weighted center of the galaxy group is marked by an open star symbol at $\left(-17.9^{\prime \prime},-19.0^{\prime \prime}\right)$ from the QSO sightline. The line-of-sight velocity map of [O II] emission is presented in the right panel with zero velocity corresponding to $z_{\mathrm{abs}}=0.6226$. The location of the LLS is marked by an open magenta circle. The galaxy group spans a range in the line-of-sight velocity offset from $d v_{\text {gal }}=-499 \mathrm{~km} \mathrm{~s}^{-1}$ to $d v_{\text {gal }}=+184$ $\mathrm{km} \mathrm{s}^{-1}$ from the absorber with a light-weighted center at $d v_{\text {gal }}=-170 \mathrm{~km} \mathrm{~s}^{-1}$ (open star symbol in panel $b$ ). While the galaxy group members all exhibit an ordinary continuum morphology in the pseudo $r$-band image, the [O II] contours revealed spatially-extended line-emitting nebulae around the two massive group members at $d v_{\text {gal }}=-129$ and $-499 \mathrm{~km} \mathrm{~s}^{-1}$ in the lower-right corner, suggesting strong interactions between the two galaxies. The galaxy at $d v_{\text {gal }}=-499 \mathrm{~km} \mathrm{~s}^{-1}$ is also the most massive member of the group with spectral features indicative of a post-starburst phase (and possibly hosting an AGN; see text for details).

\subsection{The LLS at $z_{\text {abs }}=0.6226$ toward J2135-5316} in a massive galaxy group at $d_{\text {group }}=177$ pkpc

\subsubsection{Absorption properties of the optically-thick gas}

The LLS at $z_{\text {abs }}=0.6226$ toward J2135-5316 has $\tau_{912}>6.3$. It is the second strongest LLS found in our sample (after the $\mathrm{H}_{2}$-bearing DLA toward J0111-0316), and is resolved into a minimum of six components with $\approx 99 \%$ of the $N\left(\mathrm{H}_{\mathrm{I}}\right)$ contained in the central component at $d v_{c}=0$ $\mathrm{km} \mathrm{s}^{-1}$ (component 3 in Table 6 for this system) and $\approx 1 \%$ contained in the components at $d v_{c}=-120,+26$, and +56 $\mathrm{km} \mathrm{s}^{-1}$ (components 2, 4, and 5, respectively, in Table 6). The remaining components contribute negligibly to the total $N\left(\mathrm{H}_{\mathrm{I}}\right)$, but they dominate the line width observed in the first few Lyman lines at $d v_{c}=-268 \mathrm{~km} \mathrm{~s}^{-1}$ and $d v_{c}=+98$ $\mathrm{km} \mathrm{s}^{-1}$ (Figures 3 and 4). In particular, the large $b$ value of component $6\left(b_{c} \approx 83 \mathrm{~km} \mathrm{~s}^{-1}\right.$ and $\left.\log N_{c}\left(\mathrm{H} \mathrm{I}_{\mathrm{I}}\right) / \mathrm{cm}^{-2} \approx 14.2\right)$ in Table 6 is likely due to blended weak $\mathrm{H}_{\text {I }}$ components that are not resolved in the COS spectra, but are necessary for explaining the observed $\operatorname{Ly} \alpha$ and $\operatorname{Ly} \beta$ line profiles. This component is not present in higher-order Lyman lines and the lack of resolving power has a negligible impact on the total $N\left(\mathrm{H}\right.$ I) measurement, but the best-fit $N_{c}\left(\mathrm{H}_{\mathrm{I}}\right)$ for component 6 should be taken with caution.

While low-ionization species are concentrated in the two strongest $\mathrm{H}_{\mathrm{I}}$ components at $d v_{c}=0$ and $+26 \mathrm{~km} \mathrm{~s}^{-1}$ (components $3 \& 4$ in Table 6 ), intermediate ions exhibit match- ing component structure with the first few Lyman series lines at $-120 \lesssim d v_{c} \lesssim 60 \mathrm{~km} \mathrm{~s}^{-1}$. In particular, component 2 at $d v_{c}=-120 \mathrm{~km} \mathrm{~s}^{-1}$ exhibits strong and relatively narrrow $\mathrm{C}_{\mathrm{III}}$ and $\mathrm{O}_{\mathrm{VI}}$ absorption and no detectable low-ionization transitions, showing a distinct ionization state from components $3 \& 4)$.

\subsubsection{The galaxy environment}

The MUSE observations of this field presented in $\S 2.4$ have uncovered 86 objects in the $1^{\prime} \times 1^{\prime}$ field of view with pseudo $r$-band magnitude ranging from $A B(r)=21$ to 26.6 mag, in addition to a bright star and the QSO. Robust redshift measurements are obtained for 46 of these objects (left panel of Figure 5). Comparing the photometric measurements of common objects observed in both MUSE and DES shows that the pseudo $r$-band magnitudes are consistent with DES $r$-band measurements to within 0.1 magnitude uncertainties. The spectroscopic survey is $100 \%,>90 \%$, and $75 \%$ complete for objects brighter than $A B(r)=23$, 24 , and $25 \mathrm{mag}$, respectively. Redshift measurements are made using a $\chi^{2}$ fitting routine that compares the observed spectrum with models formed from a linear combination of four eigenspectra from the SDSS at different redshifts (see Chen \& Mulchaey 2009 and Johnson et al. 2013 for a detailed description). The best-fit redshift of each object was visually inspected for confirmation. The redshifts of spec- 


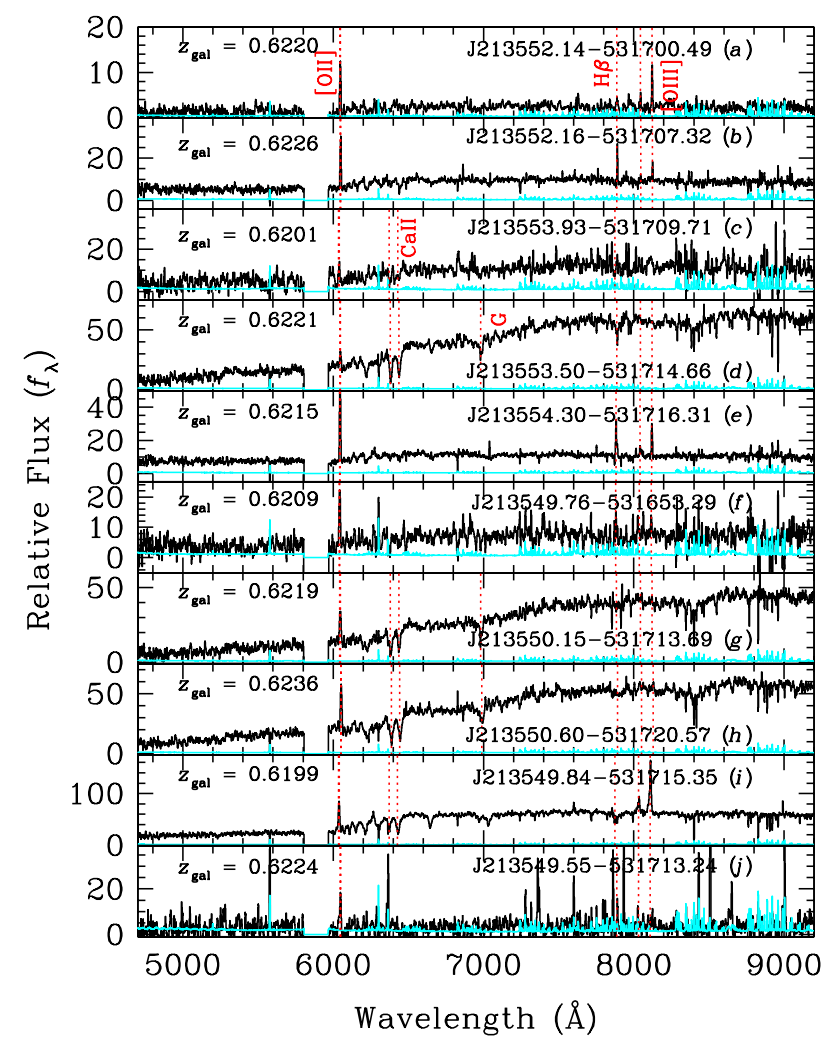

Figure 6. Optical spectra of a group of 10 galaxies identified in the vicinity of the LLS at $z_{\text {abs }}=0.6226$ toward J2135-5258 from MUSE observations. The corresponding 1- $\sigma$ error spectra are shown in cyan. The spectra are ordered with increasing projected distance of the galaxies from $d=72 \mathrm{pkpc}$ at the top to $d=252$ pkpc at the bottom. Galaxies in the group exhibit a wide range in star formation history with the most luminous and massive members displaying prominent absorption features such as Ca II and G-band (panels $d, g$, and $h$ ), indicative of evolved old stars, one galaxy displaying a strong Balmer absorption series with weak $\mathrm{H} \beta$ and [O II] (panel $i$ ), indicative of a post starburst phase, and the rest displaying strong [O II] emission and no significant Ca II absorption (panels $a, b, e, f$, and $j$ ), indicating a predominantly young stellar population. The galaxy at $z_{\text {gal }}=0.6199($ panel $i$ ) also exhibits [Ne III] and a high $[\mathrm{O} \mathrm{III}] / \mathrm{H} \beta$ line ratio, suggesting the presence of an AGN.

troscopically identified galaxies range from $z_{\text {gal }}=0.24$ to $z=4.49$

A group of 10 galaxies is found in the vicinity of the LLS with pseudo $r$-band magnitude ranging from $A B(r)=21.2$ to $24.7 \mathrm{mag}$ and line-of-sight velocity offset ranging from $d v_{\text {gal }}=-499 \mathrm{~km} \mathrm{~s}^{-1}$ to $d v_{\text {gal }}=+184 \mathrm{~km} \mathrm{~s}^{-1}$ (Figure 5). The remaining spectroscopically-identified galaxies in the MUSE field all appear at a cosmologically distinct redshift with a velocity separation exceeding $\left|d v_{\text {gal }}\right|=600 \mathrm{~km} \mathrm{~s}^{-1}$ from the LLS. The galaxies in the LLS-associated group span a range in angular distance from $\theta=10.6^{\prime \prime}$ to $\theta=38.1^{\prime \prime}$, corresponding to a range in projected distance from $d=72$ to $252 \mathrm{pkpc}$ at the redshift of the LLS. Optical spectra of the members of the LLS-associated galaxy group are presented in Figure 6 with increasing projected distance from top to bottom, which clearly show a wide range in the star formation histories among the members of the galaxy group. A detailed analysis of galaxy properties is presented in Appendix
A. In summary, we estimate its intrinsic $r$-band absolute magnitude $M_{r}$ based on the observed $A B(r)$ and find that the group galaxies span a range in $M_{r}$ from $M_{r}=-22.5$ (or $3 L_{*}$ ) for a post starburst/AGN at $d=244$ pkpc (see Appendix A) to $M_{r}=-18.3$ (or $0.06 L_{*}$ ) for a faint dwarf at $d=252 \mathrm{pkpc}$ and $d v_{\text {gal }}=-36 \mathrm{~km} \mathrm{~s}^{-1}$. In addition, we estimate $M_{\text {star }}$ based on the rest-frame $g-r$ color inferred from the MUSE spectra and the prescription of Johnson et al. (2015), and find $\log M_{\text {star }} / \mathrm{M}_{\odot}=8.6-11.2$ for these galaxies. The observed and derived properties of the galaxies, including best-fit redshift $z_{\text {gal }}$, projected distance $d$, mean line-of-sight velocity offset $\left|d v_{\text {gal }}\right|$, pseudo $r$-band magnitude $A B(r)$, intrinsic absolute $r$-band magnitude $M_{r}$, inferred total stellar mass $M_{\text {star }}$, galaxy type, The total integrated [O II] line flux, and unobscured SFR are summarized in columns (2) through (10) of Table 7.

The available MUSE data show that while the closest galaxy to the LLS is a $0.12 L_{*}$ galaxy with $M_{\text {star }} \approx$ $7.9 \times 10^{8} \mathrm{M}_{\odot}$ at $d=72 \mathrm{pkpc}$ and $d v_{\text {gal }}=-111 \mathrm{~km} \mathrm{~s}^{-1}$, it is likely part of a dynamic group containing several members that are 100 times more massive at $<200$ pkpc away. All 10 galaxies contribute to a total stellar mass of $M_{\text {star }} \approx 3.4 \times 10^{11} \mathrm{M}_{\odot}$ with $\approx 60 \%$ contained in the interacting pair. We calculate a light-weighted center located at $\left(-17.9^{\prime \prime},-19.0^{\prime \prime}\right)$ from the QSO sightline (open star symbol in Figure 5). The corresponding projected distance of the 'group' is therefore $d_{\text {group }}=177 \mathrm{pkpc}$ and the light-weighted line-of-sight velocity offset is $d v_{\text {group }} \approx-170 \mathrm{~km} \mathrm{~s}^{-1}$ between the galaxy 'group' and the LLS. We also calculate a line-of-sight velocity dispersion of $\sigma_{\text {group }}=211 \mathrm{~km} \mathrm{~s}^{-1}$, implying a dynamical mass of $M_{\text {dyn }} \approx 1.1 \times 10^{13} \mathrm{M}_{\odot}$ and a virial radius of $r_{\mathrm{vir}} \approx 360 \mathrm{kpc}$ for the galaxy group. The strong LLS may be due to gaseous streams at $\gtrsim 0.5 r_{\text {vir }}$ in the intragroup medium (e.g., Chen et al. 2019b).

\subsection{The LLS at $z_{\text {abs }}=0.5427$ toward J2308-5258 near a star-forming galaxy at $d=32$ pkpc}

\subsubsection{Absorption properties of the optically-thick gas}

The LLS at $z_{\mathrm{abs}}=0.5427$ toward $\mathrm{J} 2308-5258$ has $\tau_{912}=2.53 \pm 0.01$. It is resolved into a minimum of six components with $\approx 74 \%$ of $N(\mathrm{HI})$ contained in the central component (component 4 in Table 6) at $d v_{c}=0 \mathrm{~km} \mathrm{~s}^{-1}$ and $\approx 21 \%$ contained in the two components at $d v_{c}=-24$ and $+24 \mathrm{~km} \mathrm{~s}^{-1}$ (components 3 and 5, respectively, in Table 6). Different from previous systems, the remaining components, in particular components 1 and 6 , contribute an appreciable amount ( $3 \%$ and $2 \%$, respectively) to the total $N\left(\mathrm{H}_{\mathrm{I}}\right)$ (Figures 3 and 4 ). However, the large $b$ value of component $2\left(b_{c} \approx 60 \mathrm{~km} \mathrm{~s}^{-1}\right.$ and $\left.\log N_{c}(\mathrm{HI}) / \mathrm{cm}^{-2} \approx 15.4\right)$ in Table 6 is likely due to unresolved $\mathrm{H}_{\mathrm{I}}$ components in the COS spectra, but is necessary for explaining the observed $\operatorname{Ly} \alpha$ and Ly $\beta$ line profiles. Because this component is not present in higher-order Lyman lines, the lack of resolving power has a negligible impact $(<1 \%)$ on the total $N\left(\mathrm{HI}_{\mathrm{I}}\right)$ measurement but the best-fit $N_{c}\left(\mathrm{HI}_{\mathrm{I}}\right)$ for component 2 should be taken with caution.

There is a good one-to-one correspondence between the associated low-ionization transitions and the $\mathrm{H}$ i components with the only exception being component 2 , the broad component at $d v_{c} \approx-114 \mathrm{~km} \mathrm{~s}^{-1}$. The kinematic profiles of 
Table 7. Intrinsic properties of galaxies in the vicinity of a LLS

\begin{tabular}{|c|c|c|c|c|c|c|c|c|c|c|}
\hline $\begin{array}{l}\text { Galaxy } \\
\quad(1)\end{array}$ & $\begin{array}{l}z_{\text {gal }} \\
(2)\end{array}$ & $\begin{array}{c}d \\
(\mathrm{pkpc}) \\
(3)\end{array}$ & $\begin{array}{c}d v_{\mathrm{gal}}^{a} \\
(\mathrm{~km} / \mathrm{s}) \\
(4)\end{array}$ & $\begin{array}{c}A B(r) \\
(5)\end{array}$ & $\begin{array}{c}M_{r}{ }^{b} \\
(6)\end{array}$ & $\begin{array}{c}\log M_{\text {star }} / \mathrm{M}_{\odot} \\
(7)\end{array}$ & $\begin{array}{c}\text { Type }^{c} \\
(8)\end{array}$ & $\begin{array}{c}f_{\text {line }}{ }^{d} \\
\left(10^{-17} \mathrm{erg} / \mathrm{s} / \mathrm{cm}^{2}\right) \\
(9)\end{array}$ & $\begin{array}{c}\mathrm{SFR}^{e} \\
\left(\mathrm{M}_{\odot} / \mathrm{yr}\right) \\
(10)\end{array}$ & $\begin{array}{c}\text { ISM } \\
(\mathrm{O} / \mathrm{H})^{f} \\
(11)\end{array}$ \\
\hline \multicolumn{11}{|c|}{ Galaxies associated with the LLS at $z_{\text {abs }}=0.6226$ toward J2135-5258 } \\
\hline $\mathrm{J} 213552.14-531700.49$ & 0.6220 & 72 & -111 & 24.0 & -19.0 & 8.9 & $\mathrm{SF}$ & $2.06 \pm 0.03$ & 0.22 & $\ldots$ \\
\hline $\mathrm{J} 213552.16-531707.32$ & 0.6226 & 100 & 0 & 22.6 & -20.4 & 9.4 & SF & $3.77 \pm 0.04$ & 0.40 & $\ldots$ \\
\hline $\mathrm{J} 213553.93-531709.71$ & 0.6201 & 104 & -462 & 23.7 & -20.0 & 10.2 & $\mathrm{~A}$ & $0.05 \pm 0.01$ & 0.01 & $\ldots$ \\
\hline $\mathrm{J} 213553.50-531714.66$ & 0.6221 & 129 & -92 & 21.6 & -22.0 & 10.7 & A & $0.64 \pm 0.08$ & 0.07 & $\ldots$ \\
\hline $\mathrm{J} 213554.30-531716.31$ & 0.6215 & 154 & -203 & 22.3 & -20.7 & 9.6 & SF & $1.14 \pm 0.05$ & 0.12 & $\ldots$ \\
\hline $\mathrm{J} 213549.76-531653.29$ & 0.6209 & 211 & -314 & 23.0 & -20.0 & 9.3 & SF & $1.00 \pm 0.03$ & 0.11 & $\ldots$ \\
\hline J213550.15-531713.69 & 0.6219 & 222 & -129 & 21.8 & -21.9 & 10.6 & $\mathrm{~A}$ & $4.96 \pm 0.16$ & 0.53 & $\ldots$ \\
\hline J213550.60-531720.57 & 0.6236 & 231 & +184 & 21.5 & -22.2 & 10.8 & A & $6.47 \pm 0.30$ & 0.69 & $\ldots$ \\
\hline J213549.84-531715.35 & 0.6199 & 244 & -499 & 21.2 & -22.5 & 11.2 & PSB & $15.23 \pm 0.72$ & 1.63 & $\ldots$ \\
\hline J213549.55-531713.24 & 0.6224 & 252 & -36 & 24.7 & -18.3 & 8.6 & $\mathrm{SF}$ & $0.26 \pm 0.02$ & 0.03 & $\ldots$ \\
\hline \multicolumn{11}{|c|}{ Galaxy associated with the LLS at $z_{\mathrm{abs}}=0.5427$ toward $\mathrm{J} 2308-5258^{g}$} \\
\hline $\mathrm{J} 230837.42-525845.07$ & 0.5426 & 32 & -19 & 21.0 & -22.1 & 10.9 & $\mathrm{SF}+\mathrm{A}$ & $\ldots$ & $\ldots$ & $\ldots$ \\
\hline \multicolumn{11}{|c|}{ Galaxies associated with the LLS at $z_{\text {abs }}=0.3640$ toward J0248-4048 } \\
\hline J024806.09-404835.64 & 0.3638 & 15 & -44 & $\ldots$ & $\ldots$ & $\ldots$ & SF & $4.41 \pm 0.03$ & 0.11 & 7.7 \\
\hline J024805.69-404831.04 & 0.3639 & 37 & -22 & 25.1 & -16.3 & 7.8 & $\mathrm{SF}$ & $0.63 \pm 0.02$ & 0.01 & 7.5 \\
\hline J024803.87-404822.24 & 0.3637 & 150 & -66 & 24.1 & -17.3 & 8.2 & $\mathrm{SF}$ & $1.98 \pm 0.02$ & 0.05 & 7.6 \\
\hline \multicolumn{11}{|c|}{ Galaxy associated with the LLS at $z_{\mathrm{abs}}=0.4353$ toward J0357-4812 } \\
\hline J035723.07-481215.78 & 0.4358 & 67 & +105 & 20.7 & -21.7 & 10.4 & $\mathrm{SF}+\mathrm{A}$ & $6.14 \pm 0.06$ & 0.28 & $\ldots$ \\
\hline \multicolumn{11}{|c|}{ 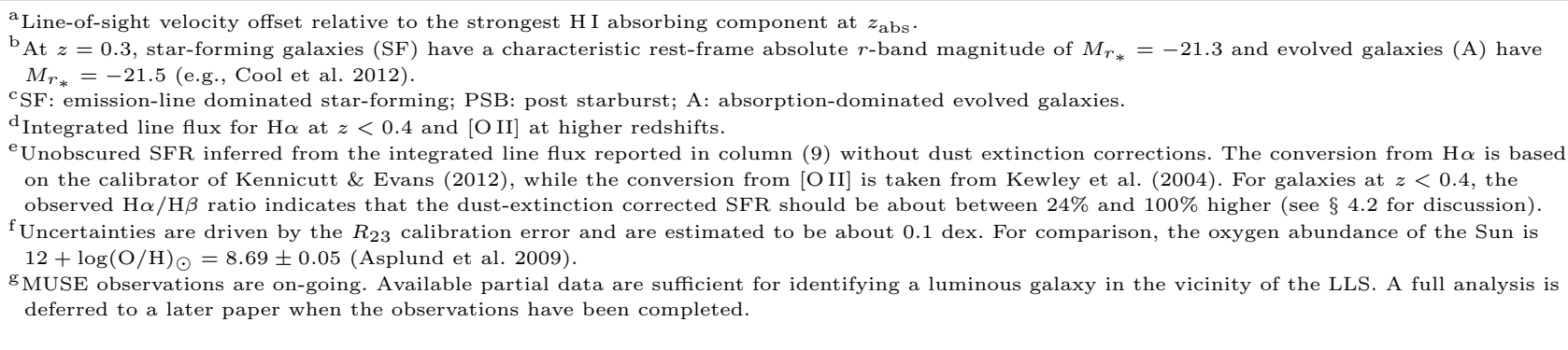 } \\
\hline
\end{tabular}

low ions also match well with those of intermediate- and highly-ionized species. However, it is clear that these components exhibit distinct $N\left(\mathrm{H}_{\mathrm{I}}\right)$ to $N(\mathrm{Mg} \mathrm{II})$ ratios. Specifically, components 1 and 3 exhibit a column density ratio of $2: 7$ in $N\left(\mathrm{H}_{\mathrm{I}}\right)$, while the associated $\mathrm{Mg}$ II components exhibit a comparable strength. The differential $N\left(\mathrm{Mg}_{\mathrm{II}}\right) / N\left(\mathrm{H}_{\mathrm{I}}\right)$ ratios strongly imply differences in the underlying gas metallicity between these components (e.g., Zahedy et al. 2019). Different from the LLS at $z=0.6226$ toward J2135-5316, the $\mathrm{O}_{\mathrm{VI}}$ doublet of this LLS is broad and covers the velocity range of all lower-ionization species, highlighting the multiphase nature of the gas.

\subsubsection{The galaxy environment}

The MUSE observations of this field are ongoing. Only a third of the observations have been executed. It is expected that the data will reach a comparable depth as seen in J0357-4812 (see $\S 4.5$ below). An initial analysis of the data collected so far has revealed a luminous disk galaxy of $A B(r) \approx 21 \mathrm{mag}$ at $z_{\text {gal }}=0.5426$ and $\theta=5.1^{\prime \prime}$ from the QSO sightline, corresponding to $d=32 \mathrm{pkpc}$ and $d v_{\text {gal }}=-19 \mathrm{~km} \mathrm{~s}^{-1}$ from the LLS. At $z=0.54$, the observed pseudo $r$-band magnitude implies $M_{r} \approx-22.1$ (or $2.1 L_{*}$ ) and $\log M_{\text {star }} / \mathrm{M}_{\odot} \approx 10.9$. Similar to the LLSabsorbing galaxy in J0357-4812 (see $\S 4.5$ and Figure 11), the optical spectrum of this galaxy is characterized by a combination of strong Ca II H\&K absorption and strong [O II]

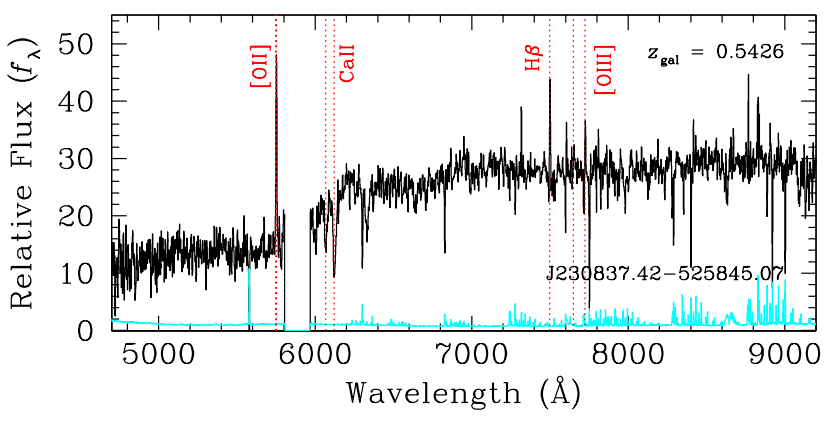

Figure 7. Optical spectrum of a luminous galaxy identified in the vicinity of the LLS at $z_{\text {abs }}=0.5427$ toward J2308-5258 from available MUSE data. The corresponding 1- $\sigma$ error spectrum is shown in cyan. The galaxy is bright and the spectrum exhibits prominent absorption features due to Ca II H\&K and the Balmer series, along with strong $[\mathrm{O} \mathrm{II}]$ and $\mathrm{H} \beta$ emission lines (highlighted in red dotted lines with corresponding line identifications).

and $\mathrm{H} \beta$ emission lines (Figure 7). Different from the configuration between the QSO probe and the LLS absorbing galaxy in J0357-4812, the QSO here probes the diffuse gas at $\approx 28^{\circ}$ from the minor axis of the inclined disk. While no other galaxies are found in the vicinity of the LLS to $A B(r) \approx 25.5 \mathrm{mag}$, suggesting that the luminous galaxy at $d=32 \mathrm{pkpc}$ is singularly responsible for the LLS, the survey 


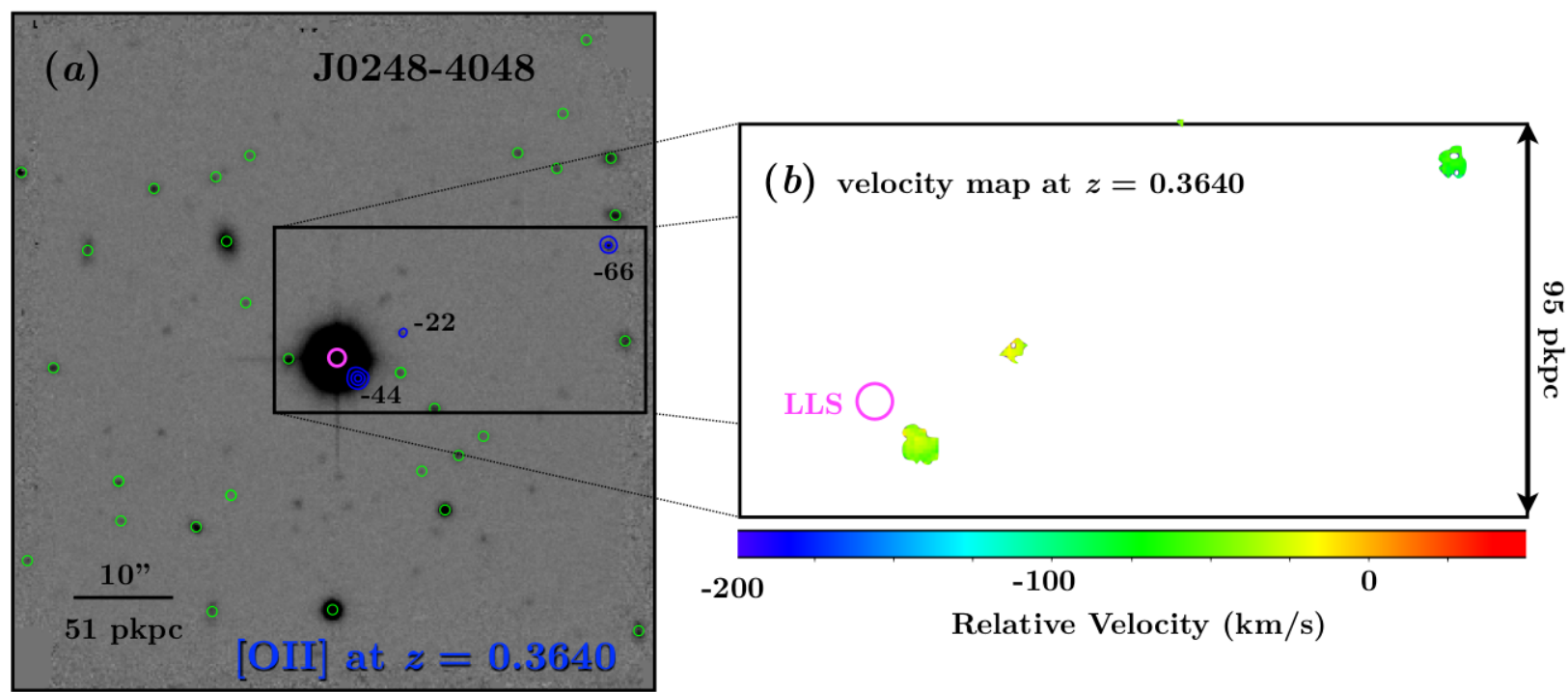

Figure 8. The galaxy environment uncovered by MUSE for the LLS at $z_{\mathrm{abs}}=0.3640$ toward J0248-4048. The pseudo $r$-band image from Figure 2 is reproduced in panel $(a)$. North is up and east is to the left. Objects that are spectroscopically identified at a cosmologically distinct redshift from the LLS are marked by green circles, while galaxies associated with the LLS are highlighted by blue [O II] emission contours of constant surface brightness $0.25,1.25$, and $2.5 \times 10^{-17} \mathrm{erg} \mathrm{s}^{-1} \mathrm{~cm}^{-2} \operatorname{arcsec}^{-2}$ and marked by the line-of-sight velocity offset $\left(\mathrm{km} \mathrm{s}^{-1}\right)$ from $z_{\mathrm{abs}}=0.3640$. Three galaxies are found in the vicinity of the LLS with angular distance of $\theta=3.0^{\prime \prime}, 7.8^{\prime \prime}$, and $29.7^{\prime \prime}$ from the QSO sightline (magenta circle), corresponding to $d=15,37$, and $150 \mathrm{pkpc}$, respectively, at the redshift of the LLS. The line-of-sight velocity map based on a simultaneous fit to the observed [O II] doublet, $\mathrm{H} \beta$, [O III] $\lambda \lambda$ 4960, 5008, and $\mathrm{H} \alpha$ (see Figure 9) is presented in the right panel with zero velocity corresponding to $z_{\mathrm{abs}}=0.3640$. The location of the LLS is marked by an open magenta circle. All three LLS-associated galaxies are found at relatively small line-of-sight velocity offset of $|\Delta v|<70 \mathrm{~km} \mathrm{~s}^{-1}$ from the absorber.

is still incomplete. A detailed analysis of the galactic environment of this LLS will be presented in a later paper when complete galaxy survey data are available from MUSE and Magellan.

\subsection{The LLS at $z_{\text {abs }}=0.3640$ toward J0248-4048 near a pair of low-mass dwarfs at $d \approx 26$ pkpc}

\subsubsection{Absorption properties of the optically-thick gas}

The LLS at $z_{\text {abs }}=0.3640$ toward J0248-4048 has $\tau_{912}=2.48 \pm 0.01$. It is resolved into a minimum of six components with $\approx 87 \%$ of $N\left(\mathrm{H}_{\mathrm{I}}\right)$ contained in the central component at $d v_{c}=0 \mathrm{~km} \mathrm{~s}^{-1}$ and $\approx 12 \%$ contained in the component at $d v_{c}=-42 \mathrm{~km} \mathrm{~s}^{-1}$ (components 3 and 2 , respectively, in Table 6). The remaining components contribute no more than $1 \%$ to the total $N\left(\mathrm{H}_{\mathrm{I}}\right)$, but they dominate the line width observed in the first few Lyman lines with velocity ranging from $d v_{c}=-131 \mathrm{~km} \mathrm{~s}^{-1}$ to $d v_{c}=+154$ $\mathrm{km} \mathrm{s}^{-1}$ (Figures 3 and 4 ). The large $b$-value of component $5\left(b_{c} \approx 69 \mathrm{~km} \mathrm{~s}^{-1}\right.$ and $\left.\log N_{c}\left(\mathrm{H}_{\mathrm{I}}\right) / \mathrm{cm}^{-2} \approx 14.7\right)$ in Table 6 is likely due to blended weak $\mathrm{H}_{\text {I }}$ components that are not resolved in the COS spectra, but is necessary for explaining the observed $\operatorname{Ly} \alpha$ and $\operatorname{Ly} \beta$ line profiles. Similar to component 6 of the LLS toward J2135-5316, this component is not present in higher-order Lyman lines and the lack of resolving power has a negligible impact on the total $N\left(\mathrm{H}_{\mathrm{I}}\right)$ measurement. However, the best-fit $N_{c}(\mathrm{H}$ I) for component 5 should be taken with caution.

While low-ionization species are concentrated in the two strongest $\mathrm{H}_{\mathrm{I}}$ components at $d v_{c}=-42$ and $0 \mathrm{~km} \mathrm{~s}^{-1}$ (components 2 and 3 in Table 6 ), intermediate ions exhibit a matching component structure with the first few Lyman series lines. In addition, the highly ionized species traced by the $\mathrm{O}$ vi doublet exhibit a broader line profile encompassing all lower-ionization species, revealing a multiphase nature of the LLS.

\subsubsection{The galaxy environment}

The MUSE observations of this field presented in $\S 2.4$ provide a deep view of the line-of-sight galaxy properties. We have uncovered 67 objects in the MUSE field of view with pseudo $r$-band magnitude ranging from $A B(r)=20.5$ to $26.9 \mathrm{mag}$ in addition to the QSO. Of the 30 objects brighter than $A B(r)=25 \mathrm{mag}$, we are able to determine a robust redshift for 28 , reaching a survey completeness of $>90 \%$. The redshifts of spectroscopically identified galaxies in the MUSE field around J0248-4048 range from $z_{\text {gal }}=0.21$ to $z_{\text {gal }}=1.46$. Three galaxies are found in the vicinity of the LLS with line-of-sight velocity offset $\left|d v_{\text {gal }}\right|<70 \mathrm{~km} \mathrm{~s}^{-1}$ (Figure 8). The remaining spectroscopically-identified galaxies in the MUSE field all appear at a cosmologically distinct redshift with a velocity separation exceeding $\left|d v_{\text {gal }}\right|=1000$ $\mathrm{km} \mathrm{s}^{-1}$ from the LLS. The angular distances of the three LLS-associated galaxies are $\theta=3.0^{\prime \prime}, 7.8^{\prime \prime}$, and $29.7^{\prime \prime}$, corresponding to $d=15,37$, and $150 \mathrm{pkpc}$, respectively, at the redshift of the LLS.

The extracted MUSE spectra of the three LLSassociated galaxies are presented in Figure 9 with increasing projected distance from top to bottom. All three galaxies exhibit strong nebular emission lines, including [O II] $\mathrm{H} \beta$, [O III], and $\mathrm{H} \alpha$, commonly seen in star-forming regions, and they are of low mass with faint traces of continuum emission. 


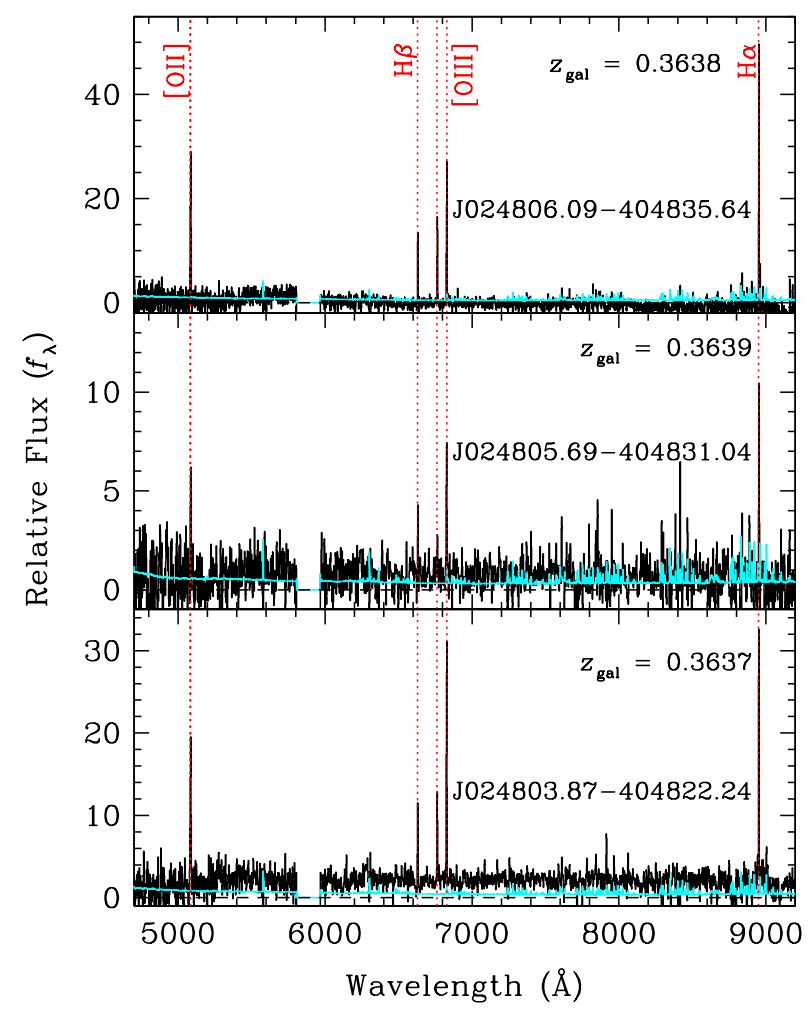

Figure 9. Optical spectra of three galaxies identified in the vicinity of the LLS at $z_{\text {abs }}=0.3640$ toward J0248 -4048 from MUSE observations. The corresponding 1- $\sigma$ error spectra are shown in cyan. The spectra are ordered with increasing projected distance from top to bottom, at $d=15,37$, and $150 \mathrm{pkpc}$, from the QSO sightline. All three galaxies exhibit an emission-line dominated spectrum. Prominent spectral features are highlighted in red dotted lines, marked with the corresponding line identifications.

Details regarding the properties of these low-mass dwarfs are presented in Appendix B. In summary, the galaxies have low luminosity, $\lesssim 0.03 L_{*}$, and low mass, $\log M_{\text {star }} / \mathrm{M}_{\odot} \lesssim 8.2$, with an unobscured SFR in the range of $0.01-0.11 \mathrm{M}_{\odot} \mathrm{yr}^{-1}$ and a gas-phase metallicity in their interstellar medium (ISM) of $\approx 10 \%$ solar. The observed and derived properties of the galaxies are shown in columns (2) through (11) of Table 7.

While both the relative line-of-sight velocity offsets and projected separations of the three low-mass dwarf galaxies are small with $\left|d v_{\text {gal }}\right|<50 \mathrm{~km} \mathrm{~s}^{-1}$ and $d \lesssim 150 \mathrm{pkpc}$ to the LLS, the observed low stellar mass also implies a low-mass host dark matter halo. If no massive galaxies are found in the ongoing shallow and wide survey beyond the MUSE field of view, then the low-mass dwarf at $d=150$ pkpc may be too far away to be sharing a common dark matter halo with the two galaxies at $d<40 \mathrm{pkpc}$. Indeed, adopting a range of the stellar mass to dark matter halo mass ratio for low-mass dwarfs from Miller et al. (2014) and Read et al. (2017), we infer a dark matter halo mass of $\log M_{h} \approx 9.8-10.6$ for the galaxy at $d=150 \mathrm{pkpc}$ and a corresponding virial radius of $r_{\mathrm{vir}} \approx 35-65 \mathrm{kpc}$. The LLS along the QSO sightline would be at a distance more than $2 \times r_{\text {vir }}$ from the galaxy at $d=150$ pkpc. We therefore conclude that the LLS is most likely associated with a pair of low-mass dwarfs at $d=15$ and 37 pkpc, respectively, with an ISM metallicity of $\approx 10 \%$ solar (see $\S 5.3$ below for further discussion).

\subsection{The LLS at $z_{\text {abs }}=0.4353$ toward J0357-4812 near a rotating disk galaxy at $d=67$ pkpc}

\subsubsection{Absorption properties of the optically-thick gas}

The LLS at $z_{\text {abs }}=0.4353$ toward J0357-4812 has $\tau_{912}=0.99 \pm 0.01$. It is resolved into a minimum of six components with $\gtrsim 99 \%$ of $N\left(\mathrm{H}_{\mathrm{I}}\right)$ contained in the central three components at $\left|d v_{c}\right|<40 \mathrm{~km} \mathrm{~s}^{-1}$ (components 1 through 3 in Table 6). The remaining components contribute negligibly to the total $N\left(\mathrm{H}_{\mathrm{I}}\right)$, but are necessary for explaining the observed line profiles of the first few Lyman lines with velocity ranging from $d v_{c}=86 \mathrm{~km} \mathrm{~s}^{-1}$ to $d v_{c}=+252 \mathrm{~km} \mathrm{~s}^{-1}$ (Figures 3 and 4 ).

Figure 4 shows that the component at $d v_{c}=+31.4$ $\mathrm{km} \mathrm{s}^{-1}$ (component 3 of this LLS in Table 6) exhibits the strongest metal absorption, while containing only $\approx 8 \%$ of the total $N\left(\mathrm{H}_{\mathrm{I}}\right)$. Specifically, components 2 and 3 exhibit a column density ratio of 10:1 in $N\left(\mathrm{H}_{\mathrm{I}}\right)$, but $4: 9$ in $N(\mathrm{Mg}$ II $)$. This differential absorption strength between $\mathrm{H}$ I and associated ionic transitions of components 2 and 3 applies to all ions observed from low- to high-ionization species, strongly implying a difference in the underlying gas metallicity (e.g., Prochter et al. 2010; Zahedy et al. 2019). Similar to the LLSs toward J2308-5258 and J0248-4048, the highly ionized species traced by the $\mathrm{O}$ vi doublet exhibit a broad line profile encompassing all lower-ionization species, indicating a multiphase nature of the LLS.

\subsubsection{The galaxy environment}

The MUSE observations of this field presented in $\S 2.4$ have uncovered 129 objects in the MUSE field of view with pseudo $r$-band magnitude ranging from $A B(r)=20.5$ to 27 mag in addition to the QSO. Of the 64 objects brighter than $A B(r)=25 \mathrm{mag}$, we are able to determine a robust redshift for 51 , reaching a survey completeness of $80 \%$. The redshifts of spectroscopically identified galaxies in this field range from $z_{\text {gal }}=0.20$ to $z_{\text {gal }}=3.668$. Similar to the LLS toward J2308-5258 (see $\S 4.3$ ), only one galaxy is found in the vicinity of the LLS at line-of-sight velocity offset $d v_{\text {gal }}=+105$ $\mathrm{km} \mathrm{s}^{-1}$ and angular distance $\theta=11.9^{\prime \prime}$, corresponding to $d=67 \mathrm{pkpc}$ at the redshift of the LLS (Figure 10). Different from the configuration between the QSO probe and the LLS absorbing galaxy in J2308-5258, the QSO here probes the diffuse gas at $\approx 27^{\circ}$ from the major axis of the inclined disk (see below). The remaining spectroscopically-identified galaxies in the MUSE field are all at a cosmologically distinct redshift with a velocity separation exceeding $\left|d v_{\text {gal }}\right|=1000$ $\mathrm{km} \mathrm{s}^{-1}$ from the LLS. The observed $r$-band magnitude of the LLS-absorbing galaxy is $A B(r)=20.7$, while objects without a robust redshift measurement are all fainter than $A B(r)=23.3 \mathrm{mag}$. Any additional galaxy in the vicinity of the LLS will have to be more than 10 times fainter with intrinsic luminosity $<0.14 L_{*}$.

The optical spectrum of the absorbing galaxy at $d=67$ pkpc is presented in Figure 11, which is characterized by strong absorption features, together with weak $[\mathrm{O}$ II $]$ and $\mathrm{H} \beta$ emission lines. In particular, it exhibits prominent $\mathrm{Ca}$ II absorption along with strong Balmer absorption lines. Details regarding the properties of this galaxy are presented in Appendix C. In summary, the galaxy is luminous with an unobscured $\mathrm{SFR}$ of $\approx 0.28 \mathrm{M}_{\odot} \mathrm{yr}^{-1}$ based on the total integrated 

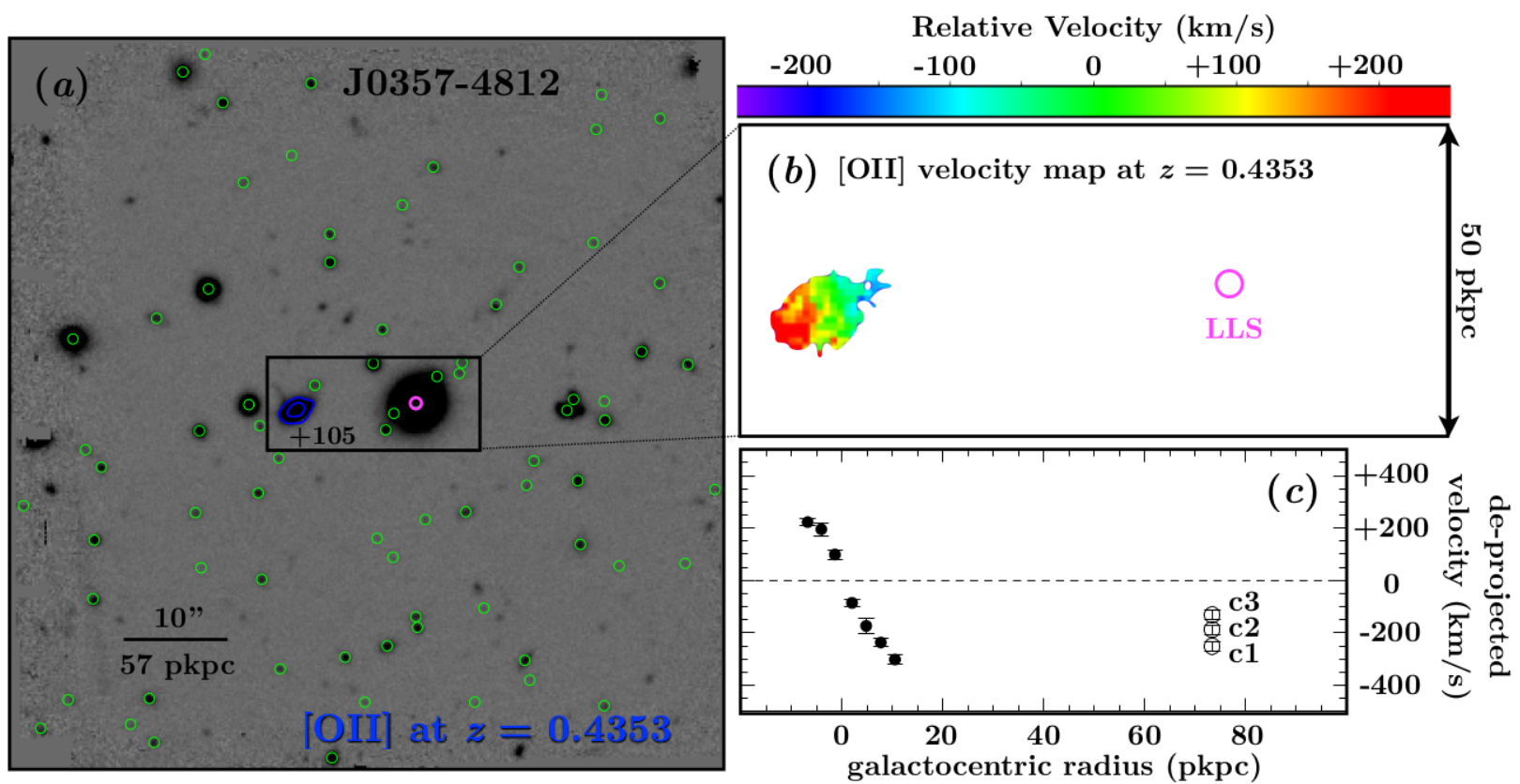

Figure 10. The galaxy environment uncovered by MUSE for the LLS at $z_{\text {abs }}=0.4353$ toward J0357-4812. The pseudo $r$-band image from Figure 2 is reproduced in panel $(a)$. North is up and east is to the left. Of the 77 spectroscopically identified galaxies in the MUSE field of view (green circles), only one galaxy (highlighted in blue contours) is found in the vicinity of the LLS at $\theta=11.9^{\prime \prime}$ (or $d=67$

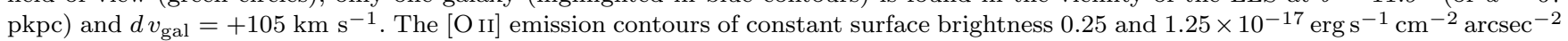
are superimposed on top of the pseudo $r$-band image. The line-of-sight velocity map of [O II] emission is presented in panel (b) with zero velocity corresponding to $z_{\mathrm{abs}}=0.4353$. The location of the LLS is marked by an open magenta circle. The absorbing galaxy displays a clear velocity gradient along the long axis, supporting the presence of a rotating disk. Panel (c) shows the de-projected rotation velocity along the disk plane as a function of galactocentric radius (solid points). Here zero velocity is shifted from the LLS to the systemic redshift of the galaxy. For comparison, the three strongest H I absorbing components (c1, c2, and c3 from Table 6) of the LLS along the QSO sightline are also included in the plot, under the assumption that the gas is moving along the plane extended from the inclined optical disk. The de-projected velocities of the three components are consistent with a general rotation motion with the galaxy (see text for further discussion).

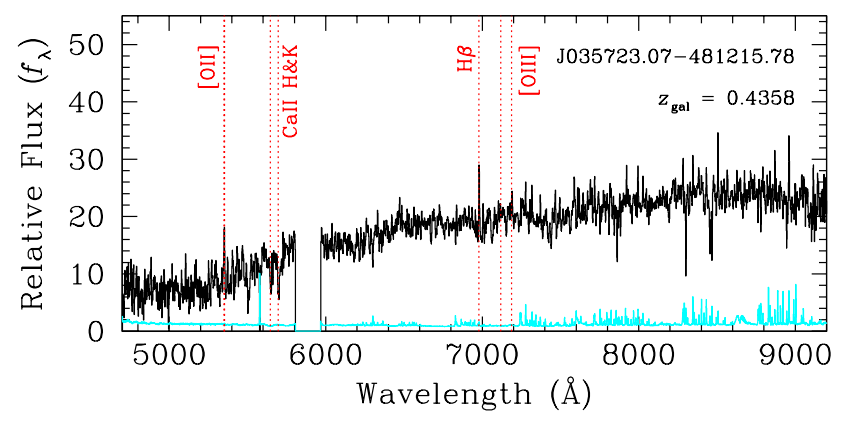

Figure 11. Optical spectrum of the galaxy identified in the vicinity of the LLS at $z_{\text {abs }}=0.4353$ toward J0357-4812 from MUSE observations. The corresponding 1- $\sigma$ error spectrum is shown in cyan. The galaxy is bright and the spectrum exhibits prominent absorption features along with weak [O II] and $\mathrm{H} \beta$ emission lines (highlighted in red dotted lines with corresponding line identifications). In addition to the Ca II absorption doublet, the spectrum also exhibits strong Balmer absorption lines, indicating the presence of mixed stellar populations.

[O II] line flux and the star formation calibrator of Kewley et al. (2004). The observed $r$-band magnitude, $A B(r)=20.7$, and rest-frame $g-r$ color translate to an intrinsic $r$-band absolute magnitude of $M_{r}=-21.7$ (corresponding to $1.4 L_{*}$ ) based on an early-type disk galaxy template and an underlying stellar mass of $\log M_{\text {star }} / \mathrm{M}_{\odot}=10.4$. The observed and derived properties of the galaxy are presented in columns (2) through (11) of Table 7.

The line-of-sight velocity map presented in panel $(b)$ of Figure 10 shows that the absorbing galaxy displays a clear velocity gradient along the long axis, from $\approx+250 \mathrm{~km} \mathrm{~s}^{-1}$ on the southeast (lower-left) side to $\approx-110 \mathrm{~km} \mathrm{~s}^{-1}$ on the northwest (upper-right) side of the galaxy with the zero velocity corresponding to the systemic redshift, $z=0.4353$, of the strongest component (c2 in Table 6 ) in the LLS at $d=67$ pkpc to the west. The de-projected rotation velocity along the disk plane as a function of galactocentric radius is presented in panel (c) of Figure 10 (solid points) with zero velocity corresponding to the systemic redshift of the galaxy (see Appendix C for details). Adopting $d v_{\text {deproj }} \approx+225 \mathrm{~km} \mathrm{~s}^{-1}$ to be the maximum rotation velocity would imply an underlying dark matter halo mass of $M_{h} \approx 10^{12} \mathrm{M}_{\odot}$ and a stellar mass to dark matter halo mass ratio of $M_{\text {star }} / M_{h} \approx 2.5 \%$. The three $\mathrm{H}_{\mathrm{I}}$ absorbing components containing $>99 \%$ of the total $N\left(\mathrm{HI}_{\mathrm{I}}\right)(\mathrm{c} 1, \mathrm{c} 2$, and $\mathrm{c} 3$ from Table 6 ) of the LLS are found to be moving along the same direction as the optical disk at a velocity, ranging from $d v_{\text {deproj }}($ LLS $)=-130$ to $-250 \mathrm{~km} \mathrm{~s}^{-1}$ (open circles in panel $c$ of Figure 10). The observed co-rotation between the gas and the galaxy suggests that the LLS originates in a rotating halo or an ex- 
tended disk plane (e.g., Steidel et al. 2002; Chen et al. 2005; Diamond-Stanic et al. 2016; Martin et al. 2019) with contributions from either extraplanar gas and/or high velocity clouds (e.g., Heald et al. 2011). Interestingly, component 3 with the largest $N(\mathrm{Mg}$ II $)$ (and likely higher gas metallicity; see discussion in $\S 5.2$ ) also exhibits the largest lag in $\left|d v_{\text {deproj }}\right|$ (see Figure 4$)$.

\section{DISCUSSION}

The LLS survey in the CUBS QSO sample has uncovered five new LLSs with $\tau_{912} \gtrsim 1$ at $z_{\text {abs }}=0.3640-0.6226$ and five pLLS with $\tau_{912}=0.2-1$ at $z_{\text {abs }}=0.2603-$ 0.9372 . While the number of newly identified LLSs is small, these represent a uniform and unbiased sample of opticallythick absorbers identified along random QSO sightlines with $\log N\left(\mathrm{H} \mathrm{I}_{\mathrm{I}}\right) / \mathrm{cm}^{-2} \approx 16.5$. Common features of the newly identified LLSs include: (1) multi-component kinematic profiles, indicating a clumpy medium, and (2) a simultaneous presence of ionic species in multiple ionization states, suggesting a multiphase nature of the gas. These features have important implications for studying the ionization state and metallicity of the gas.

Deep galaxy survey data in the LLS fields have revealed a diverse range of galaxy environments around these optically-thick absorbers, from a massive quiescent halo, a low-mass dwarf galaxy pair, a co-rotating gaseous halo/disk, to possibly intragroup medium of a galaxy group. A joint analysis, combining absorption-line studies of newly discovered LLSs and the associated galaxy survey data from the CUBS program, has offered important new insights into the connection between optically-thick gas and galaxies, as well as the physical origin of metal-line absorbers. In this section, we discuss the implications of our sample for the incidence of LLSs at $z<1$ and the physical properties of these absorbers, and the galactic environment uncovered from our galaxy survey.

\subsection{Incidence of Lyman Limit Systems at $z<1$}

Because of their optically thick nature, the rate of incidence of LLSs is an important quantity for constraining the mean free path of ionizing photons and for computing the IGM photo-ionization rate. It also places constraints on the covering fraction of optically-thick gas connected to infalling clouds and/or outflows in galaxy halos (e.g., Hafen et al. 2017). Previous surveys have yielded a number density of LLSs per unit redshift per sightline in the range of $n(z) \approx 0.3-0.7$ at $z<1$ (e.g., Songaila \& Cowie 2010 for a compilation of early results with updates from Ribaudo et al. 2011; Shull et al. 2017). The large uncertainty underscores the challenge in conducting LLS surveys. First, surveys for LLSs at low redshifts rely on UV spectroscopy from space and therefore require a sample of UV-bright QSOs at $z_{\mathrm{QSO}} \gtrsim 1$ as the background probes. Secondly, these systems are rare and when a LLS occurs, it also attenuates the background QSO light and further reduces the survey path and sensitivity for uncovering additional Lyman limit features at lower redshift. This is seen in two of the CUBS QSO sightlines in Table 5, J0111-0316 and J2135-5316, which exhibit

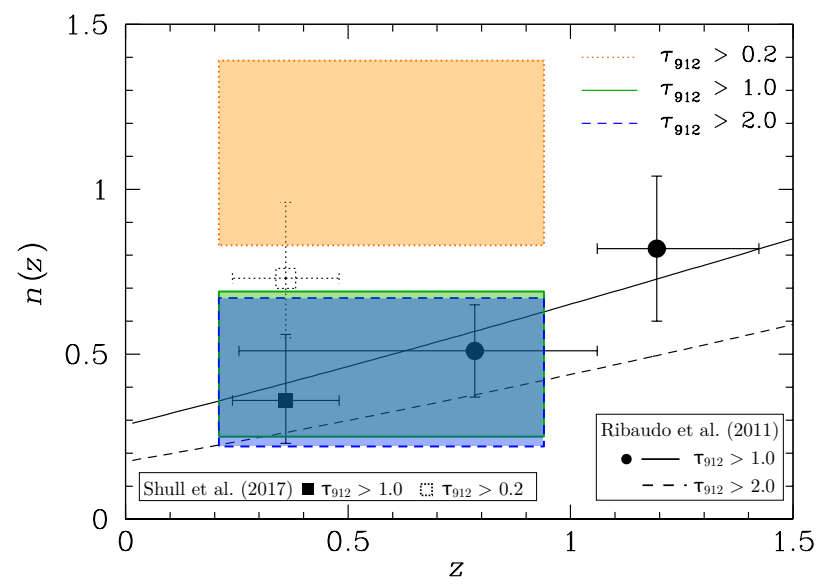

Figure 12. The rate of incidence of optically-thick $\mathrm{H}$ I absorbers at $z \lesssim 1$ from the CUBS QSO sample. The measurements are made for three different $\tau_{912}$ thresholds, from $\tau_{912}>2$ (blue, dashed rectangle), $\tau_{912}>1$ (green, solid rectangle), to $\tau_{912}>0.2$ (orange, dotted rectangle). The height of each rectangle represents the $68 \%$ confidence interval estimated from a bootstrap resampling exercise described in the text, and the width represents the redshift interval surveyed. Previous measurements and associated 1- $\sigma$ uncertainties from Ribaudo et al. (2011) are shown in solid points, along with their best-fit redshift evolution models for $\tau_{912}>1$ (solid curve) and for $\tau_{912}>2$ (dashed curve). The measurements and 1- $\sigma$ errors from Shull et al. (2017) are shown in squares.

the two strongest LLSs found in our survey and consequently $\Delta z_{\mathrm{LL}}$ is substantially reduced for those two sightlines.

The consequence of attenuated background QSO light when a LLS occurs has important implications for the sample definition of QSOs employed for an unbiased CGM survey. UV-bright QSOs are biased against finding LLSs which, when unaccounted for, would lead to an underestimate of the incidence of these optically thick systems (e.g., Tytler 1982). Previous LLS surveys relying on archival sightlines to maximize the redshift survey pathlength for these relatively rare systems have attempted to minimize such a bias by excluding QSO sightlines that are known to be biased against LLSs (e.g., Ribaudo et al. 2011). However, the sample is inherently heterogeneous and likely contains implicit bias. A recent study of the GALEX FUV-NUV colors of a sample of over 9000 NUV-selected QSOs (see the illustration in the top panel of Figure 1) indeed suggests that the number density of LLSs at $z<1$ may have been underestimated (Deharveng et al. 2019).

The CUBS QSO sample is selected to be at $z_{\mathrm{QSO}} \approx 0.8-$ 1.4 and bright in the GALEX NUV channel (1770-2730 $\AA$ ). As outlined in $\S 2$, these selection criteria are motivated by a key scientific goal of studying the inner CGM $(d \lesssim 100$ pkpc $)$ at $z \lesssim 1$ where a high incidence of optically-thick absorbers is expected. The QSO sample is therefore also optimized for an unbiased search of LLS at $z<1$. The summary in Table 5 shows that the entire CUBS QSO sample offers a total survey pathlength of $\Delta z_{\mathrm{LL}}=9.31$ for LLSs at $z=0.21$ 0.94. To estimate the number density of low-redshift LLSs and the associated uncertainties, we perform a bootstrap resampling routine. Specifically, we randomly resample the parent CUBS QSO sightlines with replacement to generate a 
new QSO sample containing the same number of QSOs. For each Lyman limit decrement in the new QSO sample, a new $\tau_{912}$ is generated from randomly sampling the Gaussian error around the measured $\tau_{912}$ reported in Table 5 . This exercise is repeated 1000 times to produce a distribution of newly estimated number density $n(z)$ of the LLSs. The resulting 16 and 84 percentiles mark the $68 \%$ confidence interval of the best estimated $n(z)$.

Our survey yields a number density estimate of $n(z)=$ $0.43_{-0.18}^{+0.26}$ for systems with $\tau_{912}>1$. This is consistent with expectations from previous archival studies by Ribaudo et al. (2011) and Shull et al. (2017). It is, however, evident from Table 5 that most of the newly discovered LLSs in the CUBS QSO sample exhibit $\tau_{912}$ significantly greater than one. We repeat the number density measurement for two different thresholds with $\tau_{912}>2$ and $\tau_{912}>0.2$. The results are presented in Figure 12, along with measurements from previous archival studies for comparison. For $\tau_{912}>2$, we find $n(z)=0.43_{-0.21}^{+0.24}$ in comparison to $n(z)=0.37 \pm 0.10$ from Ribaudo et al. (2011). For $\tau_{912}>0.2$, we find $n(z)=1.08_{-0.25}^{+0.31}$ in comparison to $n(z)=0.73_{-0.18}^{+0.23}$ from Shull et al. (2017). Our best estimated $n(z)$ for these strong $\mathrm{H}$ i absorbers are higher than previously reported values, but the uncertainties are large due to the small QSO sample.

\subsection{The Origin of Lyman Limit Systems}

We have shown in $\S 3.2$ that in order to fully characterize the Lyman series associated with each LLS, it is necessary to incorporate a multi-component structure for the absorber. Specifically, while the strongest component explains the highest-order Lyman series lines all the way to the Lyman discontinuity, additional components are needed to explain the first few Lyman lines with the largest oscillator strengths (see also Chen et al. 2018; Zahedy et al. 2019). This multi-component structure is also supported by the associated ionic transitions. In particular, nearly all $\mathrm{H}_{\mathrm{I}}$ components of $\log N\left(\mathrm{HI}_{\mathrm{I}}\right) / \mathrm{cm}^{-2}>16.8$ exhibit associated $\mathrm{Mg}$ II components that are fully resolved in the ground-based optical echelle spectra (Figure 4). While COS does not fully resolve the component structure of additional FUV lines such as $\mathrm{C}$ II, $\mathrm{C}$ III, N II, and $\mathrm{Si}$ II, there is a broad agreement in the overall line shape (including both line width and centroid) of these FUV transitions with the relative component strength displayed in the resolved $\mathrm{Mg}$ II components. The agreement in the absorption profiles between low- and intermediateionization transitions supports a scenario that these ions are linked kinematically and share a common velocity field.

We also note that $\mathrm{O}_{\mathrm{vI}}$ absorption is observed in all but the DLA at $z_{\mathrm{abs}}=0.5762$ toward J0111-0316. Three of the four O vi-bearing LLSs, toward J0248-4048, J0357-4812, and J2308-5258, display a broad and relatively shallow $\mathrm{O}$ VI absorption profile. While the component structures of the $\mathrm{O}_{\text {VI }}$ lines are not resolved, their kinematic profiles share a similar asymmetry with the associated $\mathrm{C}_{\text {III }}$ absorption line. Such agreement suggests a multiphase nature of these LLSs (see also, Zahedy et al. 2019). A notable exception is the LLS at $z_{\text {abs }}=0.6226$ toward J2135-5316, which displays a single strong $\mathrm{O}$ VI component at $d v_{c}=-120 \mathrm{~km} \mathrm{~s}^{-1}$ with corresponding $\mathrm{C}_{\mathrm{III}}$ and a relatively weak $\mathrm{H}_{\mathrm{I}}$ component of $\log N_{c}\left(\mathrm{H}_{\mathrm{I}}\right) / \mathrm{cm}^{-2}=15.4$ and $b_{c} \approx 27 \mathrm{~km} \mathrm{~s}^{-1}$, but no other low-ionization transitions. The co-presence of $\mathrm{C}_{\mathrm{III}}$ and $\mathrm{O}$ VI with consistent kinematic profiles with $\mathrm{H}$ I suggests that the gas is predominantly photo-ionized (e.g., Tripp et al. 2008; Thom \& Chen 2008; Stern et al. 2018). However, the absence of $\mathrm{O}_{\mathrm{VI}}$ around dominant $\mathrm{H}_{\mathrm{I}}$ components at $\left|d v_{c}\right|<100$ $\mathrm{km} \mathrm{s}^{-1}$ is in stark contrast to the other three LLSs toward J0248-4048, J0357-4812, and J2308-5258.

Comparisons of heavy ions to neutral hydrogen column density ratios between individual components have also revealed a chemical inhomogeneity within a single LLS. Figure 13 shows the observed $N(\mathrm{Mg}$ II $) / N\left(\mathrm{H}_{\mathrm{I}}\right)$ ratios both for individual components (pentagons) and for each LLS as a whole (horizontal bars). Only components with $\log N(\mathrm{HI}) / \mathrm{cm}^{-2}>15$ are included, because the sensitivity limit for $N(\mathrm{Mg}$ II $)$ allowed by the optical echelle spectra become unconstraining for weaker $\mathrm{H}$ i components. In at least three of the five new LLSs in the CUBS sample, large differential $N(\mathrm{Mg} \mathrm{II}) / N(\mathrm{H} \mathrm{I})$ is directly seen between different components. For example, the strongest $\mathrm{Mg}$ II component of the LLS at $z_{\text {abs }}=0.4353$ toward J0357-4812 ( $c 3$ in Table 6) occurs at $d v_{c}=+31.4 \mathrm{~km} \mathrm{~s}^{-1}$ and contains 10 times less $\mathrm{H}_{\mathrm{I}}$ than the strongest $\mathrm{H}_{\text {I }}$ component ( $c 2$ in Table 6$)$ at $d v_{c}=0 \mathrm{~km} \mathrm{~s}^{-1}$. Consequently, the two components exhibit $N(\mathrm{Mg}$ II $) / N\left(\mathrm{H}_{\mathrm{I}}\right)$ ratios that differ by more than a factor of 10 (panel $e$ of Figure 13).

Although inferring gas metallicity from the observed $N(\mathrm{Mg}$ II $) / N\left(\mathrm{H}_{\mathrm{I}}\right)$ requires knowledge of the ionization state of the gas (which is presented in a separate paper by Zahedy et al. 2020, in preparation), we note that under photoionization equilibrium the observed differences in $N_{c}(\mathrm{Mg}$ II $) / N_{c}\left(\mathrm{H}_{\mathrm{I}}\right)$ could serve as a rough guide for inferring the underlying gas metallicities for a limited gas density range under photo-ionization equilibrium. This is understood based on the expectation that the abundance ratio of total $\mathrm{Mg} / \mathrm{H}$ is related to $N(\mathrm{Mg}$ II $) / N\left(\mathrm{HI}_{\mathrm{I}}\right)$ according to $(\mathrm{Mg} / \mathrm{H}) \times\left(f_{\mathrm{Mg}^{+}} / f_{\mathrm{H}^{0}}\right)=\mathrm{Mg}^{+} / \mathrm{H}^{0}$. For a typical gas density of diffuse halo gas in the range $n_{\mathrm{H}} \approx 0.01-0.001 \mathrm{~cm}^{-3}$ (corresponding to an ionization parameter in the range of $\log U \approx-3--2$ at $z<1$, assuming ionization equilibrium and no dust depletion), the ratio of ion fractions, $f_{\mathrm{Mg}^{+}} / f_{\mathrm{H}^{0}}$, does not change by more than a factor of two (e.g., Chen $\&$ Tinker 2008; Wotta et al. 2016). The large variation by more than a factor of 10 in the observed $N(\mathrm{Mg} \mathrm{II}) / N\left(\mathrm{H}_{\mathrm{I}}\right)$ between individual components therefore suggests large differential chemical enrichment levels in individual LLSs (see also Prochter et al. 2010; Zahedy et al. 2019).

In summary, newly uncovered LLSs in the CUBS QSO sample exhibit a multi-component absorption structure with differential chemical enrichment levels as well as ionization states. The gas is complex in terms of its kinematics and ionization state, and inhomogeneous in chemical enrichment. These properties have important implications both for the physical origins of these optically-thick absorbers and for ionization and metallicity measurements that are based on integrated column density ratios over all components. They also underscore the scientific value of high-resolution absorption spectra in resolving the clumpy, multiphase gas (e.g., Zahedy et al. 2019; Rudie et al. 2019). 


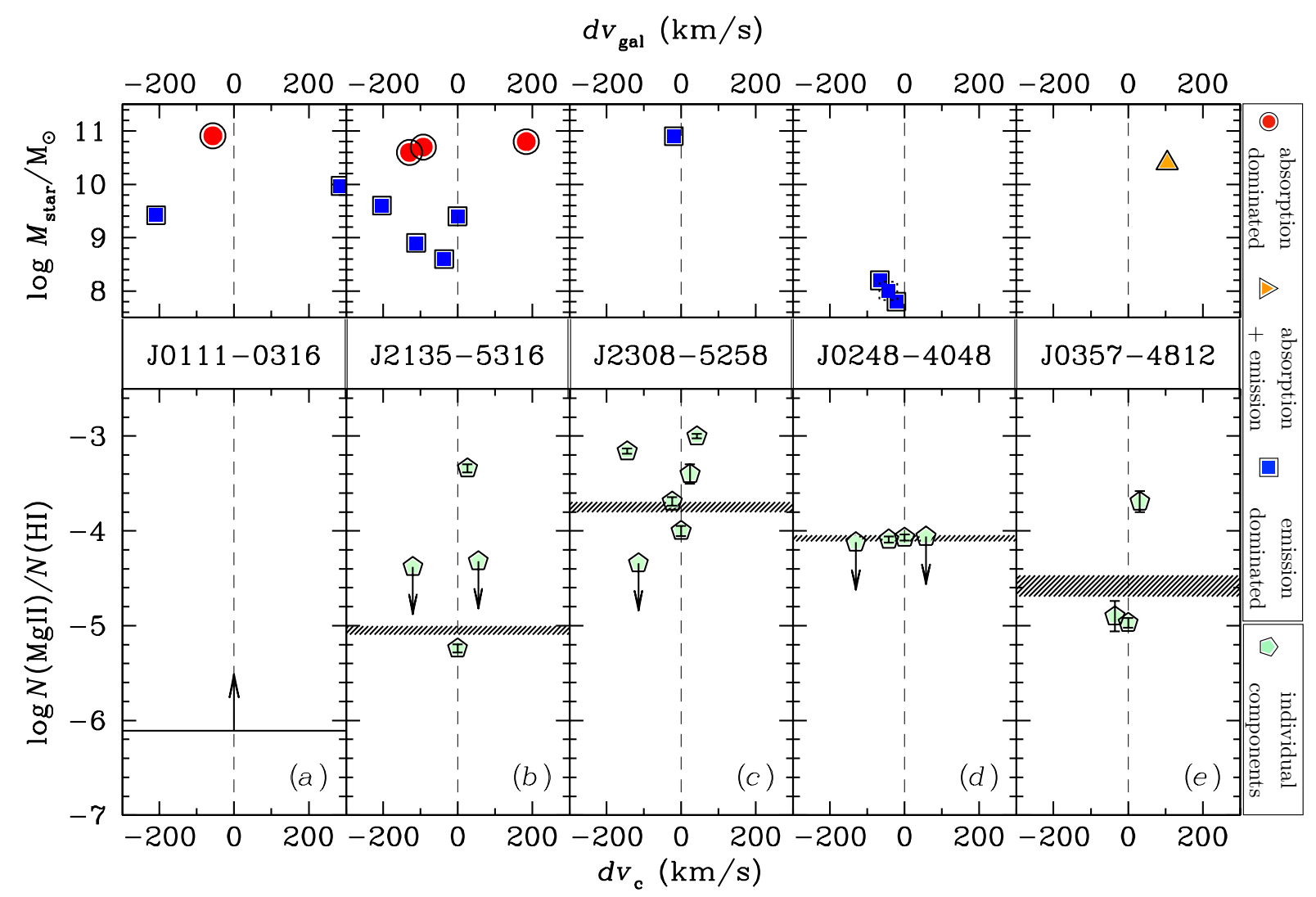

Figure 13. Top: Stellar mass versus velocity distribution of galaxies identified at $d<300 \mathrm{pkpc}$ and $\left|d v_{\text {gal }}\right|<300 \mathrm{~km} \mathrm{~s}^{-1}$ from the LLS (see Table 7). Blue squares represent emission-line dominated star-forming galaxies; red circles represent absorption-line (such as Ca II H\&K) dominated galaxies; and orange triangles represent galaxies displaying both emission and absorption in their optical spectra. The square with a dotted outline represents the galaxy at $d=15$ pkpc toward J0248-4048, because its mass is inferred from the estimated gas-phase metallicity and the mass-metallicity relation of Berg et al. (2012). Bottom: Observed $N(\mathrm{Mg}$ II $) / N(\mathrm{H}$ I) ratios of five new LLSs from the CUBS sample versus the line of sight velocity offset from the strongest H I component ( $d v_{c}$ from Table 6$)$. The fields are ordered with decreasing $N(\mathrm{H} \mathrm{I})$ from left to right. Pentagons are for individual components of $\log N(\mathrm{H} \mathrm{I}) / \mathrm{cm}^{-2}>15$, while the horizontal bar in each panel represents the integrated column density ratio and its associated uncertainty over all components for each LLS. Large scatter between individual components is present in three of the four strong $\mathrm{H}$ I absorbers for which the component structure is resolved for both $\mathrm{Mg}$ II and $\mathrm{H}_{\mathrm{I}}$ (panels $b, c$, and $e$ ), and absorbing gas with high $N(\mathrm{Mg} \mathrm{II}) / N(\mathrm{H} \mathrm{I})$ would be missed in integrated column density ratios.

\subsection{Galaxy Environment of Lyman limit Systems}

In addition to complex gas kinematics, ionization states, and chemical enrichment histories of the gas revealed in the QSO absorption spectra, the accompanying galaxy survey has also revealed a diverse range of galaxy environments around the new LLSs in the CUBS sample. To summarize the galaxy environment of the LLSs, we show in the top panels of Figure 13 the stellar mass versus velocity distribution of galaxies identified at $d<300 \mathrm{pkpc}$ and $\left|d v_{\text {gal }}\right|<300$ $\mathrm{km} \mathrm{s}^{-1}$ from the LLS. In addition, we present the $M_{\text {star }}$ versus $d$ distribution in Figure 14. It shows the properties of galaxies identified at $d<300$ pkpc of the LLSs broadly classified into three different spectral types: evolved galaxies with an absorption-line dominated spectrum (circles), intermediate galaxies displaying both nebular emission and stellar absorption features (triangles), and star-forming galaxies displaying predominantly nebular emission lines (squares). As described in $\S 4$, the MUSE data enable a highly complete galaxy survey to faint magnitudes, reaching $100 \%$ completeness at $A B(r) \leqslant 23 \mathrm{mag}$ and $\gtrsim 75 \%$ at $A B(r) \leqslant 25 \mathrm{mag}$, in the field of view that corresponds roughly to an area of $\approx 300 \mathrm{pkpc}$ in radius at the redshifts of these absorbers. The absence of additional galaxies in the vicinities of the two LLSs toward J2308-5258 (panel c) and J0357-4812 (panel e) is therefore particularly interesting. At the redshifts of these two LLSs, our galaxy survey completeness ensures that any additional galaxy in the vicinity of the LLS would be faint with intrinsic luminosity $\lesssim 0.1 L_{*}$. It indicates that the LLSs and the associated absorbing galaxies (both super- $L_{*}$ and massive) reside in a relatively isolated environment. Galaxies identified at $d<300$ pkpc of the $\mathrm{H}_{2^{-}}$ bearing DLA are included for comparison. As described in Boettcher et al. (2020), six additional galaxies are found at $\left|d v_{c}\right|<300 \mathrm{~km} \mathrm{~s}^{-1}$ at $d=300-600$ pkpc from the ongoing deep- and wide-field galaxy survey on the Magellan Telescopes. However, within the loose group of nine galaxies, the evolved galaxy at $d=42 \mathrm{pkpc}$ is the most massive member, contributing $\approx 40 \%$ of the total stellar mass of the group.

Similarly, the LLS at $z_{\text {abs }}=0.6226$ toward J2135-5316 is found associated with a massive galaxy group of dynamical mass $M_{\text {dyn }} \approx 1.1 \times 10^{13} \mathrm{M}_{\odot}$ (comparable in mass to halos hosting luminous red galaxies; Padmanabhan et al. 2007) at 


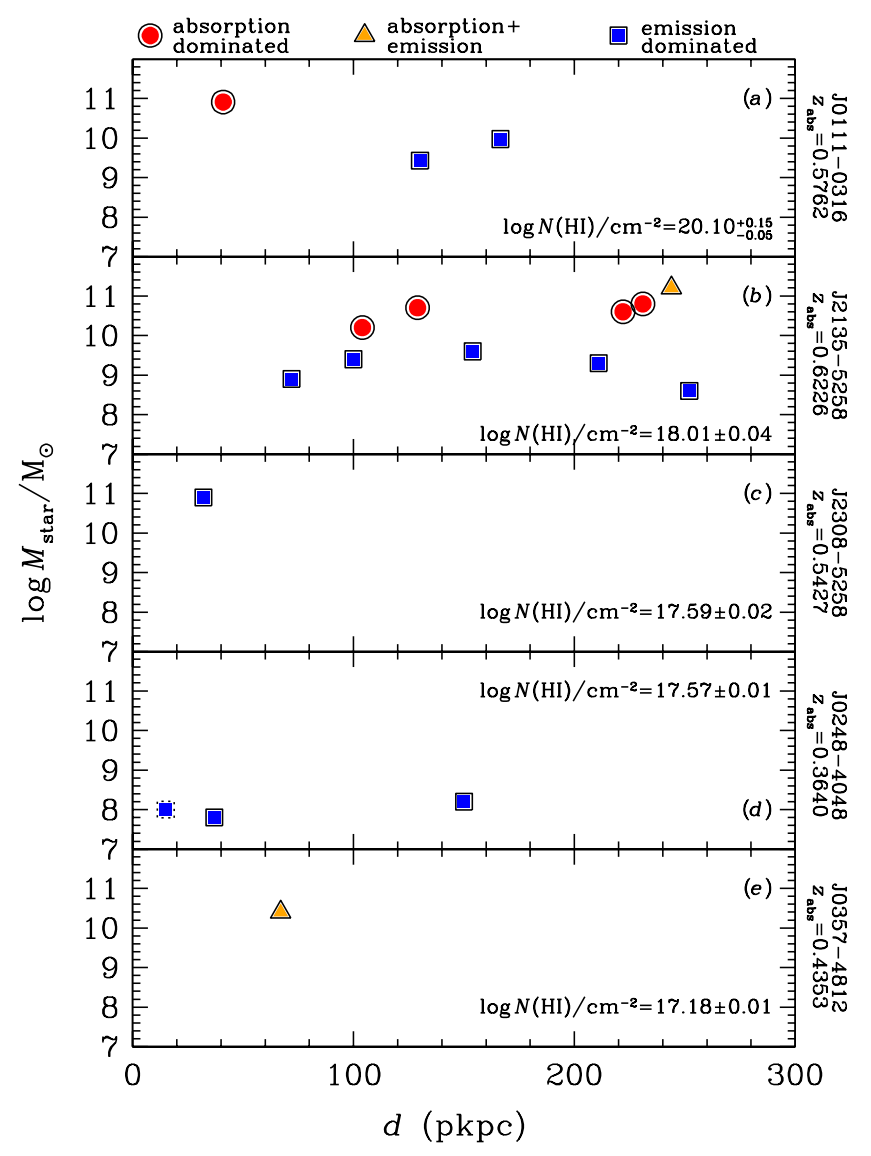

Figure 14. Summary of the galaxy environment uncovered from follow-up deep galaxy surveys of the five new optically-thick absorbers in the CUBS sample. The panels display $M_{\text {star }}$ versus $d$ for galaxies found in the vicinities of the LLSs along the sightlines toward J0111-0316 (panel $a$; from Boettcher et al. 2020), J2135-5316 (panel b), J2308-5258 (panel c), J0248-4048 (panel $d$ ), and J0357-4812 (panel $e$ ). The fields are ordered with decreasing $N(\mathrm{H} \mathrm{I})$ from top to down. Blue squares represent emission-line dominated star-forming galaxies; red circles represent absorptionline (such as CaII H\&K) dominated galaxies; and orange triangles represent galaxies displaying both emission and absorption in their optical spectra. The galaxy at $d=15 \mathrm{pkpc}$ toward J0248-4048 is included for comparison (shown in dotted outline in panel $d$ ), but note that, as discussed in $\S 4.4$, the mass is inferred from the estimated gas-phase metallicity and the massmetallicity relation of Berg et al. (2012). MUSE data enable a galaxy survey to faint magnitudes, reaching $100 \%$ completeness at $A B(r) \leqslant 23 \mathrm{mag}$ and $\gtrsim 75 \%$ at $A B(r) \leqslant 25 \mathrm{mag}$, in the field of view that corresponds roughly to an area of $\approx 300 \mathrm{pkpc}$ in radius at the redshifts of these absorbers. The absence of additional galaxies in the vicinities of the two LLSs toward J0357-4812 and J2308-5258 therefore indicates that the LLSs and the associated galaxies (both are super- $L_{*}$ ) reside in a relatively isolated environment.

$d_{\text {group }}=177$ pkpc. The massive group environment provides a potential physical explanation for the lack of $\mathrm{O}_{\mathrm{VI}}$ surrounding the strong $\mathrm{H}_{\mathrm{I}}$ components (see Figure 4). Because the gas temperature in a massive group is expected to be high, $T \sim 6 \times 10^{6} \mathrm{~K}$, heavy elements, including oxygen, are expected to be ionized to higher states (e.g., Oppenheimer et al. 2016). Indeed, Wijers et al. (2020) predict covering frac- tions of only $\approx 25 \%$ for $\mathrm{O}$ VI gas of $\log N(\mathrm{O}$ vI $) / \mathrm{cm}^{-2}>13.5$ at $d \approx 200$ pkpc from a galaxy with $\log M_{\text {star }} / \mathrm{M}_{\odot}>11$. At the same time, the likely photo-ionized $\mathrm{O}$ VI component at $d v_{c}=-120 \mathrm{~km} \mathrm{~s}^{-1}$ with $\log N(\mathrm{HI}) / \mathrm{cm}^{-2}=15.4$ may be attributed to halo clouds associated with the nearby lowmass satellite of $\log M_{\text {star }} / \mathrm{M}_{\odot}=8.9$ at $d=72 \mathrm{pkpc}$ and $\Delta v=-111 \mathrm{~km} \mathrm{~s}^{-1}$. However, the dominant $\mathrm{H}_{\mathrm{I}}$ component of this LLS contains the second highest $N(\mathrm{HI})$ uncovered in our survey with $\log N(\mathrm{HI}) / \mathrm{cm}^{-2}=18$ and only relatively weak $\mathrm{Mg}$ II absorption. The low $N(\mathrm{Mg}$ II $) / N\left(\mathrm{H}_{\mathrm{I}}\right)$ ratio (panel $b$ of Figure 13) implies a relatively low gas metallicity. It is therefore likely that the LLS originate in dense gaseous streams accreted from the IGM (e.g., Hafen et al. 2017).

In contrast to the two strongest $\mathrm{H}_{\mathrm{I}}$ absorbers uncovered in our survey, the LLS at $z_{\text {abs }}=0.3640$ toward J0248-4048 is found to be associated with a pair of low-mass dwarfs of $\approx 0.01-0.03 L_{*}\left(\log M_{\mathrm{star}} / \mathrm{M}_{\odot} \approx 8\right)$ and ISM metallicity of $\approx 10 \%$ solar at $d=15$ and $37 \mathrm{pkpc}$. The galaxy at $d=15 \mathrm{pkpc}$ is the closest absorbing galaxy uncovered in our survey. However, the total $\mathrm{H}_{\mathrm{I}}$ column density is merely $\log N(\mathrm{HI}) / \mathrm{cm}^{-2}=17.6$. This low $N(\mathrm{HI})$ at a small distance from a low-mass dwarf is similar to what is observed in local dwarf irregular galaxies (e.g., Hunter et al. 2012; Johnson et al. 2017). The low-mass nature (and presumably shallow gravitational potential well) in the presence of the UV background makes it difficult to maintain a large amount of neutral gas (e.g., Johnson et al. 2017). The two dominant $\mathrm{H}_{\mathrm{I}}$ components at $d v_{c}=-42$ and $d v_{c}=0$ $\mathrm{km} \mathrm{s}^{-1}(c 2$ and $c 3)$ of this LLS exhibit comparably modest $N_{c}(\mathrm{Mg}$ II $) / N_{c}\left(\mathrm{H}_{\mathrm{I}}\right)$ ratios (panel $d$ of Figure 13), suggesting a chemical enrichment level comparable to the $10 \%$ solar metallicity (e.g., Wotta et al. 2016) inferred for the ISM of the two star-forming dwarfs. If confirmed by a more detailed ionization analysis, the optically-thick gas most likely originates in expelled ISM gas from the nearby dwarf galaxies, either through galaxy interactions or starburst driven outflows.

Both the LLSs toward J0357-4812 and J2308-5258 are found in a relatively isolated environment with only one luminous, star-forming galaxy found at $d<300 \mathrm{pkpc}$ and $\left|d v_{\text {gal }}\right|<300 \mathrm{~km} \mathrm{~s}^{-1}$. Incorporating results from ongoing deep- and wide-field galaxy survey will provide a more complete view of the larger-scale galaxy environment. In the immediate vicinity of the LLS, however, the LLS at $z_{\text {abs }}=0.4353$ toward J0357-4812 is found to be associated with a luminous galaxy of $\approx 1.4 L_{*}$ at $d=67 \mathrm{pkpc}$ with the QSO sightline intercepting the diffuse halo at $\approx 27^{\circ}$ from the major axis (see $\S 4.5$ ). The relative velocity offsets of the absorbing components are consistent with the gas co-rotating with the disk, with the high-metallicity component ( $c 3)$ showing the largest "lag" in velocity. If the low-metallicity components $(c 1$ and $c 2$ ) originate in accreted gaseous streams that co-rotate, possible origins for the highmetallicity component $(c 3)$ include fountain flows, extraplanar gas, or disrupted satellites (see Heald et al. 2011 and Putman et al. 2012 for a review).

In contrast, the LLS at $z_{\text {abs }}=0.5427$ toward J2308-5258 is found to be associated with a luminous, starforming galaxy of $\approx 2 L_{*}$ at $d=32 \mathrm{pkpc}$ with the QSO sightline intercepting the diffuse halo at $\approx 28^{\circ}$ from the minor axis (see $\S 4.3$ ). The $\mathrm{Mg}$ II absorption profile is resolved into five separate components with four satellite components at 
$\left|d v_{c}\right|>0(c 1, c 3, c 5$, and $c 6)$ sharing comparable absorption strength, while the corresponding $N(\mathrm{HI})$ differ by a factor of $\approx 6$. Following the same argument described above, the different $N_{c}(\mathrm{Mg}$ II $) / N_{c}\left(\mathrm{H}_{\mathrm{I}}\right)$ ratios suggest that the two outlying components at $d v_{c}=-144$ and $+42 \mathrm{~km} \mathrm{~s}^{-1}, c 1$ and $c 6$, respectively, contain the highest-metallicity gas in this LLS (panel $c$ in Figure 13). The combination of a close proximity to a star-forming galaxy and a geometric alignment of the QSO sightline near the minor axis of the galaxy makes starburst outflows an attractive scenario for the LLS (e.g., Heckman et al. 1990; Shen et al. 2013). However, the large scatter in gas metallicity inferred from $N_{c}(\mathrm{Mg}$ II $) / N_{c}\left(\mathrm{H}_{\mathrm{I}}\right)$ between different components also suggests that additional sources are needed to fully explain all the components seen in the LLS, particularly the central component $(c 4)$ that dominates the total $N\left(\mathrm{H}_{\mathrm{I}}\right)$ (e.g., Hafen et al. 2019).

In summary, our galaxy survey is sensitive for detecting galaxies fainter than $0.1 L_{*}$ at $d \lesssim 300 \mathrm{kpc}$ from the LLSs. A diverse range of galaxy properties is seen around these optically-thick absorbers, from a low-mass dwarf galaxy pair, a co-rotating gaseous halo/disk, a star-forming galaxy, a massive quiescent halo, to a galaxy group. The closest galaxies found for the LLSs have projected distance ranging from $d=16$ pkpc to $d=72 \mathrm{pkpc}$ and intrinsic luminosity from $\approx 0.01 L_{*}\left(\right.$ or $\left.\log M_{\text {star }} / \mathrm{M}_{\odot}=7.8\right)$ to $\approx 3 L_{*}$ (or $\left.\log M_{\text {star }} / \mathrm{M}_{\odot}=10.9\right)$. This wide range of galaxy properties uncovered for the sample of five LLSs encompasses what is seen previously for individual systems at $z_{\text {abs }}<1$ (e.g., Kacprzak et al. 2010; Neeleman et al. 2016; Péroux et al. 2017; Chen et al. 2019a), highlighting the complex origins of these absorbers.

While no clear correlation is seen between the observed overdensity of galaxies and the kinematic spread of lowionization species, the observed absorption strengths of lowionization transitions appear to correlate with the projected distance of the closest galaxy found. Specifically, both LLSs with weak $\mathrm{Mg}$ II features of $\log N(\mathrm{Mg}$ II $) / \mathrm{cm}^{-2}<13$ (along the sightlines toward J2135-5316 and J0357-4812) have the closest galaxy found at $d>60 \mathrm{pkpc}$, whereas the remaining three have $\log N(\mathrm{Mg}$ II $) / \mathrm{cm}^{-2}>13.4$ and a galaxy found at $d<50$ pkpc. The observed trend is consistent with previous findings that the distance to a galaxy is a more dominant factor than the mass of the galaxies in driving the observed Mg II absorption strength (e.g., Chen et al. 2010a,b).

\section{SUMMARY AND CONCLUSIONS}

We present initial results from the CUBS program, designed to map diffuse baryonic structures at $z \lesssim 1$, using absorption-line spectroscopy of $15 \mathrm{UV}$ bright QSOs with matching deep galaxy survey data. CUBS QSOs are selected based on their NUV brightness to avoid biases against LLSs at $z_{\text {abs }}<1$, which are expected to attenuate the background QSO light in the FUV spectral window. Combining high-quality HST COS FUV absorption spectra with optical echelle spectra of the QSOs and deep galaxy survey data of the QSO fields obtained from the ground, we carry out a joint study of LLSs and their associated galaxies to gain insight into the connection between optically-thick gas and galaxies, as well as the physical origin of metal-line absorbers. Our findings are summarized below:
(1) The COS spectra of the 15 NUV bright QSOs in the CUBS sample provide a total redshift survey pathlength of $\Delta z_{L L}=9.3$ for searching for LLSs. We report five new LLSs of $\log N(\mathrm{HI}) / \mathrm{cm}^{-2} \gtrsim 17.2$ and five new pLLS of $\log N\left(\mathrm{H}_{\mathrm{I}}\right) / \mathrm{cm}^{-2}=16.5-17.2$, leading to a number density of $n(z)=0.43_{-0.18}^{+0.26}$ for LLS and $n(z)=1.08_{-0.25}^{+0.31}$ for pLLS and LLS combined at $z_{\text {abs }}<1$. While the uncertainties are large due to the small QSO sample, there is a tantalizing indication that previously reported $n(z)$ of these strong $\mathrm{H}_{\mathrm{I}}$ absorbers may have been underestimated, possibly due to FUV selection bias.

(2) All newly uncovered LLSs exhibit a multicomponent structure and associated heavy ions from low, intermediate-, to high-ionization states. There is broad agreement in the overall line profiles of both low- and intermediate-ionization species, indicating that different ionic species are linked kinematically and share a common velocity field. At the same time, $\mathrm{O}_{\mathrm{vI}}$ is detected in all but the DLA at $z_{\text {abs }}=0.5762$ toward J0111-0316. Three of the four O vi-bearing optically-thick absorbers display a broad and relatively shallow absorption profile that traces the broad kinematic properties of associated low- and intermediate-ionization species, while the 4th LLS displays only a single strong $\mathrm{O}$ VI in one $\mathrm{H}_{\mathrm{I}}$ component that contains $\approx 0.2 \%$ of the total $N(\mathrm{HI})$.

(3) Large differential $N(\mathrm{Mg} \mathrm{II}) / N\left(\mathrm{H}_{\mathrm{I}}\right)$ ratios, by more than a factor of 10 , are directly seen across different components of individual LLSs, suggesting inhomogeneous chemical enrichment as well as ionization state in individual galaxy halos. A detailed ionization analysis presented in Zahedy et al. (2020) will quantify the extent of density and metallicity fluctuations in individual systems. Here we show that high $N\left(\mathrm{Mg}_{\mathrm{II}}\right) / N\left(\mathrm{H}_{\mathrm{I}}\right)$ components would be missed in integrated column density calculations, which underscores the scientific value of high-resolution absorption spectra in resolving the clumpy, multiphase gas.

(4) MUSE integral field spectroscopic data, covering a $1^{\prime} \times 1^{\prime}$ field of view, has provided an ultra-deep view of the environment around three of the five new LLSs. The galaxy survey in each field is $100 \%$ complete to $A B(r)=23$ mag (corresponding to $0.3 L_{*}$ or fainter at the redshifts of the LLSs), and between $75 \%$ and $90 \%$ complete to $A B(r)=25$ mag. A diverse range of galaxy environments is seen around these LLSs, from a massive quiescent halo, a low-mass dwarf galaxy pair, a co-rotating gaseous halo/disk, to a massive galaxy group. The closest galaxies found for the LLSs have projected distance ranging from $d=16$ to $d=72 \mathrm{pkpc}$ and intrinsic luminosity from $\approx 0.01 L_{*}$ to $\approx 3 L_{*}$. The wide range of galaxy environments further underscores the complexity in connecting gas to galaxies and the need for deep galaxy survey data to fully understand the origin of absorption-line systems.

In conclusion, we are constructing a legacy data set through the CUBS program to enable comprehensive studies of the co-evolution of galaxies and their surrounding gas at $z \lesssim 1$. While the combined space-based UV and ground-based optical echelle absorption spectroscopy of distant QSOs provides a powerful tool for resolving the ionization states and chemical enrichment histories of the diffuse CGM/IGM over a broad range in gas density along 15 independent sightlines, the accompanying deep and wide galaxy survey data provide a sensitive record of the galaxy environment on both large 
and small scales. In addition to detailed ionization analyses of individual high- $N(\mathrm{HI})$ absorbers presented in Boettcher et al. (2020), Cooper et al. (2020), Johnson et al. (2020), and Zahedy et al. (2020), in future papers we will probe the physical conditions, chemical composition, and kinematics of halo gas (at $d \lesssim 300 \mathrm{pkpc}$ ) around galaxies with diverse star formation histories and examine the physical origin of different absorption-line systems based on their large-scale $(\approx 1-10 \mathrm{pMpc})$ clustering amplitudes with known galaxies. Together with published samples at low and high redshift, we will also quantify the evolution of the CGM across 10 Gyrs of cosmic evolution, with the CUBS data charting the critical period when the star formation history of the universe underwent its precipitous decline.

\section{ACKNOWLEDGMENTS}

We thank an anonymous referee for timely and constructive comments that helped improve the presentation of the paper. We thank Ruari Mackenzie for prompt and helpful advice and guidance on MUSE data reduction, and Matteo Fossati for sharing the KUBEVIZ code and assistance on analyzing the MUSE data cubes. We thank Dan Kelson for his expert assistance on reducing the galaxy survey data from the Magellan Telescopes. HWC, EB, and MCC acknowledge partial support from HST-GO15163.001A and NSF AST-1715692 grants. TC and GCR acknowledge support from HST-GO-15163.015A. SC gratefully acknowledges support from Swiss National Science Foundation grant PP00P2_163824. SDJ acknowledges support from a NASA Hubble Fellowship (HST-HF2-51375.001A). KLC acknowledges partial support from NSF AST1615296. CAFG was supported by NSF through grants AST-1517491, AST-1715216, and CAREER award AST1652522, by NASA through grant 17-ATP17-0067, by STScI through grants HST-GO-14681.011, HST-GO-14268.022-A, and HST-AR-14293.001-A, and by a Cottrell Scholar Award from the Research Corporation for Science Advancement. SL was funded by project FONDECYT 1191232. This work is based on observations made with ESO Telescopes at the Paranal Observatory under programme ID 0104.A-0147(A), observations made with the $6.5 \mathrm{~m}$ Magellan Telescopes located at Las Campanas Observatory, and spectroscopic data gathered under the HST-GO-15163.01A program using the NASA/ESA Hubble Space Telescope operated by the Space Telescope Science Institute and the Association of Universities for Research in Astronomy, Inc., under NASA contract NAS 5-26555. This research has made use of NASA's Astrophysics Data System and the NASA/IPAC Extragalactic Database (NED) which is operated by the Jet Propulsion Laboratory, California Institute of Technology, under contract with the National Aeronautics and Space Administration.

\section{DATA AVAILABILITY}

The data underlying this article will be shared on reasonable request to the corresponding author.

\section{REFERENCES}

Afruni, Andrea, Fraternali, Filippo, Pezzulli, Gabriele 2019, A\&A, 625, 11

Bacon, R., Accardo, M, Adjali, L. et al. 2010, in Groundbased and Airborne Instrumentation for Astronomy III. p. 773508

Baldwin, J. A., Phillips, M. M., Terlevich, R. (1981), PASP, 93,5

Berg, Danielle A. et al. 2012, ApJ, 754, 98

Bernstein, R., Shectman, S. A., Gunnels, S. M., Mochnacki, S., \& Athey, A. E. 2003, Proc. SPIE, 4841, 1694

Bielby, R., Crighton, N. H. M., Fumagalli, M., Morris, S. L., Stott, J. P., Tejos, N., Cantalupo, S. 2017, MNRAS, 468, 1373

Burchett, Joseph N. et al. 2019, ApJ, 877, L20

Calzetti, D. 1997, in AIP Conf. Ser. 408, The Ultraviolet Universe at Low andHigh Redshift, ed. W. H. Walle (Melville, NY: AIP),403

Calzetti, Daniela, Armus, Lee, Bohlin, Ralph C., Kinney, Anne L., Koornneef, Jan, \& Storchi-Bergmann, Thaisa 2000, ApJ, 533, 682

Cantalupo, Sebastiano et al. 2019, MNRAS, 483, 5188

Chabrier, G. 2003, PASP, 115, 763

Chen, Hsiao-Wen, Lanzetta, Kenneth M., Webb, John K., \& Barcons, Xavier 1998, ApJ, 498, 77

Chen, Hsiao-Wen, Lanzetta, Kenneth M., Webb, John K., \& Barcons, Xavier 2001, ApJ, 559, 654

Chen, Hsiao-Wen, Kennicutt, Robert C., Jr., \& Rauch, Michael 2005, ApJ, 620, 703

Chen, Hsiao-Wen and Tinker, Jeremy L. 2008, ApJ, 687, 745

Chen, Hsiao-Wen \& Mulchaey, John S. 2009, ApJ, 701, 1219

Chen H.-W., Helsby J. E., Gauthier J.-R., Shectman S. A., Thompson I. B., Tinker J. L., 2010a, ApJ, 714, 1521

Chen, H.-W., Wild, V., Tinker, J. L., Gauthier, J.-R., Helsby, J. E., Shectman, S. A., \& Thompson, I. B. 2010b, ApJ, 724, L176

Chen, Hsiao-Wen, Gauthier, Jean-René, Sharon, Keren et al. 2014, MNRAS, 438, 1435

Chen, Hsiao-Wen 2017, Outskirts of Galaxies, 434, 291

Chen, Hsiao-Wen, Zahedy, Fakhri S., Johnson, Sean D., Pierce, Rebecca M., Huang, Yun-Hsin, Weiner, Benjamin J., \& Gauthier, Jean-René 2018, MNRAS, 479, 2547

Chen, Hsiao-Wen et al. 2019a, MNRAS, 484, 431

Chen, Hsiao-Wen et al. 2019b, ApJ, 878, L33

Cool, R. J. et al. 2012, ApJ, 748, 10

Deharveng, J. -M., Milliard, B., Péroux, C., Small, T. 2019, A\&A, 623, 149

Diamond-Stanic, Aleksandar M., Coil, Alison L., Moustakas, John, Tremonti, Christy A., Sell, Paul H., Mendez, Alexander J., Hickox, Ryan C., \& Rudnick, Greg H. 2016, ApJ, 824, 24

Dressler, Alan, Hare, Tyson, Bigelow, Bruce C., \& Osip, David J. 2006, Proc. SPIE,6269, 13

Drlica-Wagner, A. \& DES Collaboration 2018, ApJS, 235, 2

Dubois, Yohan, Peirani, Sébastien, Pichon, Christophe, Devriendt, Julien, Gavazzi, Raphaël, Welker, Charlotte, \& Volonteri, Marta 2016, MNRAS, 463, 3948

Eisenstein D. J., Weinberg D. H., et al. 2011, AJ, 142, 72 
Faucher-Giguère C.-A. \& Kereš D., 2011, MNRAS, 412, L118

Faucher-Giguère, Claude-André, Hopkins, Philip F., Kereš, Dušan, Muratov, Alexander L., Quataert, Eliot, \& Murray, Norman 2015, MNRAS, 449, 987

Faucher-Giguère, C.-A., Feldmann, R., Quataert, E., et al. 2016, MNRAS, 461, L32

Faucher-Giguère, C.-A. 2020, MNRAS, 493, 1614

Foreman-Mackey, D., Hogg, D. W., Lang, D., \& Goodman, J. 2013, PASP, 125, 306

Fossati, M., Fumagalli, M., Boselli, A., Gavazzi, G., Sun, M., \& Wilman, D. J. 2016, MNRAS, 455, 2028

French, K. Decker et al. 2015, ApJ, 801, 1

Fumagalli, Michele, Prochaska, J. Xavier, Kasen, Daniel, Dekel, Avishai, Ceverino, Daniel, \& Primack, Joel R. 2011, MNRAS, 418, 1796

Gordon, Karl D., Clayton, Geoffrey C., Misselt, K. A., Landolt, Arlo U., \& Wolff, Michael J. 2003, ApJ, 594, 279

Green, J. C., Froning, C. S., Osterman, S., et al. 2012, ApJ, 744,60

Hafen, Zachary et al. 2017, MNRAS, 469, 2292

Hafen, Zachary et al. 2019, MNRAS, 488, 1248

Heald, G. et al. 2011, A\&A, 526, 118

Heckman, Timothy M., Armus, Lee, Miley, George K. 1990, ApJS, 74, 833

Hewett, Paul C. \& Wild, Vivienne 2010, MNRAS, 405, 2302

Hopkins, Philip F. et al. 2014, MNRAS, 445, 581

Hummels, Cameron B., Bryan, Greg L., Smith, Britton D., \& Turk, Matthew J. 2013, MNRAS, 430, 1548

Hunter, Deidre A. et al. 2012, AJ, 144, 134

Ji, Suoqing et al. 2019, MNRAS submitted (arXiv:1909.00003)

Johnson, S. D., Chen, H.-W., \& Mulchaey, J. S. 2013, MNRAS, 434, 1765

Johnson, S. D., Chen, H.-W., \& Mulchaey, J. S. 2015, MNRAS, 449, 3263

Johnson, Sean D., Chen, Hsiao-Wen, Mulchaey, John S., Schaye, Joop, \& Straka, Lorrie A. 2017, ApJ, 850, L10

Johnson, Sean D. et al. 2018, ApJ, 869, L1

Jones, D. H. et al. 2009, MNRAS, 399, 683

Kacprzak, Glenn G., Murphy, Michael T., Churchill, Christopher W. 2010, MNRAS, 406, 445

Kacprzak, G. G. 2017, Gas Accretion onto Galaxies, 430, 145

Kennicutt, R. C. Jr. \& Evans, N. J. 2012, ARA\&A, 50, 608 Kewley L. J., Geller M. J., \& Jansen R. A. 2004 AJ 127 2002

Lamontagne, R., Demers, S., Wesemael, F., Fontaine, G., \& Irwin, M. J. 2000, AJ, 119, 241

Lehner, Nicolas, Wotta, Christopher B., Howk, J. Christopher, O'Meara, John M., Oppenheimer, Benjamin D., Cooksey, Kathy L. 2019, ApJ, 8871

Liang, C. J., Kravtsov, A. V., \& Agertz, O. 2016, MNRAS, 458, 1164

Lofthouse, Emma K. et al. 2020, MNRAS, 491, 2057

Mackenzie, Ruari et al. 2019, MNRAS, 487, 5070

Maller A. H., Bullock J. S., 2004, MNRAS, 355, 694

Martin, Crystal L., Ho, Stephanie H., Kacprzak, Glenn G., Churchill, Christopher W. 2019, ApJ, 878, 84

Miller, Sarah H., Ellis, Richard S., Newman, Andrew B., \& Benson, Andrew 2014, ApJ, 782, 115

Monroe, TalaWanda R. et al. 2016, AJ, 152, 25
Muzahid, S., Srianand, R., \& Charlton, J. 2015, MNRAS, 448,2840

Muzahid, Sowgat, Kacprzak, Glenn G., Charlton, Jane C., \& Churchill, Christopher W. 2016, ApJ, 823, 66

Naab, T. \& Ostriker, J. P. 2017, ARA\&A, 55, 1

Neeleman, Marcel et al. 2016, ApJ, 820, 39

Nelson, Dylan, Kauffmann, Guinevere, Pillepich, Annalisa, Genel, Shy, Springel, Volker, Pakmor, Rüdiger, Hernquist, Lars, Weinberger, Rainer, Torrey, Paul, Vogelsberger, Mark, \& Marinacci, Federico 2018, MNRAS, 477, 450

Oppenheimer, B. D., Crain, R. A., \& Schaye, J. 2016, MNRAS, 460, 2157

Padmanabhan, N., Schlegel, D. J., Seljak, U., et al. 2007, MNRAS, 378, 852

Pascarelle, S. M., Lanzetta, K. M., Chen, H.-W., \& Webb, J. K. 2001, ApJ, 560, 101

Perlman, Eric S., Padovani, Paolo, Giommi, Paolo, Sambruna, Rita, Jones, Laurence R., Tzioumis, Anastasios, \& Reynolds, John 1998, AJ, 115, 1253

Péroux, Céline et al. 2017, MNRAS, 464, 2053

Péroux, C. et al. 2019, MNRAS, 485, 1595

Pettini, M. \& Pagel, B.E.J. 2004, MNRAS, 348, L59

Prochaska, J. X., Werk, J. K., Worseck, G., et al. 2017, ApJ, 837, 169

Prochter, Gabriel E., Prochaska, J. Xavier, O'Meara, John M., Burles, Scott, \& Bernstein, Rebecca A. 2010, ApJ, 708, 169

Putman, M. E., Peek, J. E. G., \& Joung, M. R. 2012, ARA\&A, 50, 491

Qu, Z. \& Bregman, J. N. 2018, ApJ, 856, 5

Read, J. I., Iorio, G., Agertz, O., \& Fraternali, F. 2017, MNRAS, 467, 2019

Ribaudo, J., Lehner, N., Howk, J. C., et al. 2011, ApJ, 743, 207

Rowlands, K., Wild, V., Nesvadba, N., Sibthorpe, B., Mortier, A., Lehnert, M., \& da Cunha, E. 2015, MNRAS, 448,258

Rudie, G. C., Steidel, C. C., Trainor, R. F., et al. 2012, ApJ, 750, 67

Rudie, Gwen C., Steidel, Charles C., Shapley, Alice E., Pettini, Max 2013, ApJ, 769, 146

Rudie, Gwen C., Newman, Andrew B., \& Murphy, Michael T. 2017, ApJ, 843, 98

Rudie, Gwen C. et al. 2019, ApJ, 885, 1

Savage, A., Browne, I. W., \& Bolton, J. G. 1976, MNRAS, 177, 77

Schaye, Joop et al. 2015, MNRAS, 446, 521

Schroetter, I. et al. 2016, ApJ, 833, 39

Shen, Sijing, Madau, Piero, Guedes, Javiera, Mayer, Lucio, Prochaska, J. Xavier, \& Wadsley, James 2013, ApJ, 765, 895

Shull, J. M., Danforth, C. W., Tilton, E. M., Moloney, J., \& Stevans, M. L. 2017, ApJ, 849, 106

Somerville, R. S. \& Davé, R. 2015, ARA\&A, 53, 51

Songaila, Antoinette and Cowie, Lennox L. 2010, ApJ, 721 1448

Steidel, C. C., Kollmeier, J. A., Shapley, A. E., Churchill, C. W., Dickinson, M., \& Pettini, M. 2002, ApJ, 570, 526 Steidel, C. C., Erb, D. K., Shapley, A. E., Pettini, M., Reddy, N., Bogosavljević, M., Rudie, G. C., \& Rakic, O. 2010, ApJ, 717, 289 
Stern, Jonathan, Faucher-Giguère, Claude-André, Hennawi, Joseph F., Hafen, Zachary, Johnson, Sean D., \& Fielding, Drummond 2018, ApJ, 865, 91

Thom, C. \& Chen, Hsiao-Wen 2008, ApJS, 179, 37

Thom, C., Tumlinson, J., Werk, J. K., et al. 2012, ApJ, 758, L41

Tripp, Todd M., Sembach, Kenneth R., Bowen, David V., Savage, Blair D., Jenkins, Edward B., Lehner, Nicolas, \& Richter, Philipp 2008, ApJS, 177, 39

Tumlinson, J., Peeples, M. S., \& Werk, J. K. 2017, ARA\&A, 55, 389

Turner, Monica L., Schaye, Joop, Steidel, Charles C., Rudie, Gwen C., \& Strom, Allison L. (2014), MNRAS, 445,1

Tytler, D. 1982, Nature, 298, 427

van de Voort, Freeke, Schaye, Joop, Altay, Gabriel, Theuns, Tom 2012, MNRAS, 421, 2809

Vogelsberger, M. et al. 2014, Nature, 509, 177

Wang, Liang, Dutton, Aaron A., Stinson, Gregory S., Macciò, Andrea V., Penzo, Camilla, Kang, Xi, Keller, Ben W., \& Wadsley, James 2015, MNRAS, 454, 83

Weilbacher P. M., Streicher O., Urrutia T., PécontalRousset A., Jarno A.Bacon R., 2014, in Manset N., Forshay P., eds, ASP Conf. Ser. Vol. 485, Astronomical Data Analysis Software and Systems XXIII. Astron. Soc.Pac., San Francisco, p. 451

Werk, Jessica K., Prochaska, J. Xavier, Thom, Christopher, Tumlinson, Jason, Tripp, Todd M., O'Meara, John M., \& Meiring, Joseph D. 2012, ApJS, 198, 3

Werk, Jessica K. et al. 2014, ApJ, 792, 8

Wijers, Nastasha A., Schaye, Joop, \& Oppenheimer, Benjamin D. 2020, MNRAS submitted (arXiv:2004.05171

Wild, Vivienne et al. 2008, MNRAS, 388, 227

Wisotzki, L., Christlieb, N., Bade, N., Beckmann, V., Köhler, T., Vanelle, C., \& Reimers, D. 2000, A\&A, 358, 77

Wotta, Christopher B., Lehner, Nicolas, Howk, J. Christopher, O'Meara, John M., \& Prochaska, J. Xavier 2016, ApJ, 831, 95

Yin, S. Y., Liang, Y. C., Hammer, F., Brinchmann, J., Zhang, B., Deng, L. C., \& Flores, H. 2007, A\&A, 462, 535 York D. G., et al. 2000, AJ, 120, 1579

Zahedy, F. S., Chen, H.-W., Rauch, M., Wilson, M. L., \& Zabludoff, A. 2016, MNRAS, 458, 2423

Zahedy, F. S., Chen, H.-W., Gauthier, J.-R., \& Rauch, M. 2017, MNRAS, 466, 1071

Zahedy, Fakhri S., Chen, Hsiao-Wen, Johnson, Sean D., Pierce, Rebecca M., Rauch, Michael, Huang, Yun-Hsin, Weiner, Benjamin J., \& Gauthier, Jean-René 2019, MNRAS, 484, 2257

Zahedy, F. S., Chen, H.-W., Boettcher, E., Rauch, M., \& Zabludoff, A. 2020, MNRAS submitted

\section{APPENDIX A: GALAXY PROPERTIES IN THE MASSIVE GROUP AT $D_{\text {GROUP }}=177$ PKPC FROM THE LLS AT $Z_{\mathrm{ABS}}=0.6226$ TOWARD J2135-5316}

Optical spectra of the members of the LLS-associated galaxy group reported in $\S 4.2 .2$ clearly show a wide range in the star formation histories among the members of the galaxy group, from young star-forming to old and evolved (Figure 6). At $z=0.6$, MUSE does not provide the spectral coverage necessary for observing $\mathrm{H} \alpha$ or $[\mathrm{N} \mathrm{II}]$. While the spectra cover higher-order Balmer transitions and other nebular lines, only [O II] emission is consistently seen among all 10 group galaxies with the Ca II H\&K absorption doublet and G-band absorption being the predominant features in five group members. In particular, the most luminous member of the group at $d=244 \mathrm{pkpc}$ and $d v_{\text {gal }}=-499 \mathrm{~km} \mathrm{~s}^{-1}$ also displays a strong Balmer absorption series in addition to Ca II absorption (panel $i$ in Figure 6), characteristic of a post-starburst phase (see French et al. 2015 and Rowlands et al. 2015 for recent references). It also shows [Ne III] and an $[\mathrm{O}$ III $] / \mathrm{H} \beta$ line ratio that suggests the presence of an active galactic nucleus (AGN).

Because of a lack of robust constraints for the dust content, we estimate an unobscured SFR based on the integrated $\left[\mathrm{O}_{\mathrm{II}}\right]$ line flux for each galaxy under the assumption that the presence of the [O $\mathrm{II}]$ lines is driven by the radiation field from young stars. To determine an integrated [O II] line flux and the velocity offset of each pixel, we employ a custom IDL code KUBEVIZ, kindly shared with us by M. Fossati, to fit the emission doublet (see Fossati et al. 2016 for a detailed description of the code). We first smooth the data cube using a $3 \times 3$ box in the image plane (corresponding to the size of the PSF) to improve the signal-to-noise $(S / N)$ per pixel without degrading the spatial resolution of the data. Then for the spectrum from each pixel, we fit the [O II] doublet using a double Gaussian function. KUBEVIZ takes into account the associated error for each spaxel in the fitting routine in order to suppress contributions from features due to sky subtraction residuals, and delivers the best-fit integrated line flux and associated error of the doublet, along with the best-fit velocity and velocity dispersion maps. We visually inspect the fitting results across the full field and repeat the fitting procedure as needed after modifying the input parameters. The total integrated $\left[\mathrm{O}_{\mathrm{II}}\right]$ line fluxes of the group galaxies range from $f_{[\mathrm{OII}]}=(5 \pm 1) \times 10^{-19} \mathrm{erg} \mathrm{s}^{-1} \mathrm{~cm}^{-2}$ to $f_{[\mathrm{OII}]}=(1.52 \pm 0.07) \times 10^{-16} \mathrm{erg} \mathrm{s}^{-1} \mathrm{~cm}^{-2}$, leading to an unobscured SFR of $\approx 0.01-1.6 \mathrm{M}_{\odot} \mathrm{yr}^{-1}$ based on the star formation calibrator of Kewley et al. (2004). The line-ofsight velocity map of the galaxy group is presented in the right panel of Figure 5.

An interesting feature of the spectra displayed in Figure 5 is the contrast between ordinary continuum morphologies of the group galaxies in the pseudo $r$-band image and the irregular morphology of [O II] line emission around two massive group members at $d v_{\text {gal }}=-129$ and $-499 \mathrm{~km} \mathrm{~s}^{-1}$ to the southwest of the LLS. The extended [ $\left.\mathrm{O}_{\mathrm{II}}\right]$ emission morphologies of the two galaxies separated by $\approx 20 \mathrm{pkpc}$ in projected distance and the large line-of-sight velocity spread from $d v \approx-500 \mathrm{~km} \mathrm{~s}^{-1}$ to $d v \approx+100 \mathrm{~km} \mathrm{~s}^{-1}$ imply that violent interactions may be taking place between the two galaxies, which may also be responsible for triggering the recent episode of star formation and possible AGN phase in the post starburst galaxy at $d v_{\text {gal }}=-499 \mathrm{~km} \mathrm{~s}^{-1}$ and $d=244$ pkpc (see e.g., Johnson et al. 2018). 


\section{APPENDIX B: LOW-MASS DWARFS AT $D \approx 26$ PKPC FROM THE LLS AT $Z_{\mathrm{ABS}}=0.3640$ TOWARD J0248-4048}

The three low-mass galaxies found in the vicinity of this LLS exhibit prominent nebular emission lines that are typical of star-forming regions (see $\S 4.4 .2$ and Figure 9). To systematically search for emission features associated with the LLS and to determine the velocity offsets, we use the KUBEVIZ software to fit line-emitting features at the redshift of the LLS in the full field covered by MUSE. We first smooth the data cube using a $3 \times 3$ box in the image plane. Then for the spectrum from each pixel, we fit all available strong emission lines (in this case, the [O II] doublet, $\mathrm{H} \beta$, $[\mathrm{O}$ III $] \lambda \lambda 4960,5008$, and $\mathrm{H} \alpha$; see Figure 9 and next paragraph) simultaneously using a Gaussian function per line and adopting a common systemic redshift and velocity width across all lines considered. KUBEVIZ outputs the best-fit integrated line flux and associated error for each line, along with the best-fit velocity and velocity dispersion maps. Finally, we visually inspect the fitting results across the full field and repeat the fitting procedure as needed after modifying the input parameters. The line-of-sight velocity map of the three LLS-associated galaxies is presented in the right panel of Figure 8. No extended line emission is detected much beyond the optical extent of the three galaxies.

The presence of prominent nebular lines in all three galaxies enable a detailed analysis of the physical properties of these LLS-associated galaxies, including the star formation rate (SFR), ionization condition, ISM gas-phase metallicity, and dust content. We first estimate an unobscured SFR based on the total integrated $\mathrm{H} \alpha$ line flux $\left(f_{\mathrm{H} \alpha}\right)$ in the MUSE data using the conversion from Kennicutt \& Evans $(2012), \mathrm{SFR}=5.37 \times 10^{-42} L_{\mathrm{H} \alpha} \mathrm{M}_{\odot} \mathrm{yr}^{-1}$, which is based on a Chabrier (2003) stellar initial mass function. The galaxies have $f_{\mathrm{H} \alpha}$ ranging from $(6.3 \pm 0.2) \times 10^{-18}$ to $(4.41 \pm 0.03) \times 10^{-17} \mathrm{erg} \mathrm{s}^{-1} \mathrm{~cm}^{-2}$, leading to an estimated SFR ranging from to 0.01 to $0.11 \mathrm{M}_{\odot} \mathrm{yr}^{-1}$ (see columns 9 and 10 of Table 7 ).

Next, we examine the ionization condition of the gas by comparing the strong line ratios, $\left[\mathrm{O}_{\mathrm{III}}\right] / \mathrm{H} \beta$ versus $[\mathrm{N}$ II $] / \mathrm{H} \alpha$. None of the three galaxies exhibit a significant [N II] line, placing a $2-\sigma$ upper limit on the $N 2$ index, $N 2 \equiv$ $\log \left[\mathrm{N}_{\mathrm{II}}\right] \lambda 6585 / \mathrm{H} \alpha$, of $N 2<-1$. In addition, the $[\mathrm{O}$ III $] / \mathrm{H} \beta$ ratio of these galaxies ranges between $\log \left[\mathrm{O}_{\mathrm{III}}\right] / \mathrm{H} \beta=0.48$ and 0.52 . Together, the observed $\left[\mathrm{O}_{\mathrm{III}}\right] / \mathrm{H} \beta$ and $\left[\mathrm{N}_{\mathrm{II}}\right] / \mathrm{H} \alpha$ ratios indicate that the ISM is ionized predominantly by young stars (e.g., Baldwin et al. 1981), rather than by active galactic nuclei (AGN).

Finally, we estimate the ISM gas-phase metallicity and dust content using common emission line calibrators. We first infer a 2- $\sigma$ upper limit to the ISM gas-phase metallicity of $12+\log (\mathrm{O} / \mathrm{H})<8.3$ based on the absence of $[\mathrm{N}$ II] and the $N 2$ calibration of Marino et al. (2013), $12+\log (\mathrm{O} / \mathrm{H})=$ $8.74+0.46 \times N 2$. Then we compute the $R_{23}$ index, defined as $R_{23} \equiv\left(\left[\mathrm{O}_{\mathrm{II}}\right] \lambda \lambda 3726,3729+\left[\mathrm{O}_{\mathrm{III}}\right] \lambda \lambda 4960,5008\right) / \mathrm{H} \beta$. For an accurate estimate of $R_{23}$, we assess the amount of dust extinction correction using the observed $\mathrm{H} \alpha / \mathrm{H} \beta$ flux ratio. Following the prescription described in Calzetti et al. $(2000)^{4}$, we estimate the color excess $E(B-V)$ accord-

4 Adopting the extinction law for the Small Magellanic Cloud ing to $E(B-V)=1.97 \log \left(\left[f_{\mathrm{H} \alpha} / f_{\mathrm{H} \beta}\right] / 2.86\right)$, and find $E(B-V)=0.15 \pm 0.01,0.23 \pm 0.04$, and $0.07 \pm 0.02$ for the galaxies at $d=15,37$, and $150 \mathrm{pkpc}$, respectively. The wavelength-dependent extinction magnitude $A(\lambda)$ is related to $E(B-V)$ following $A(\lambda)=k(\lambda) E(B-V)$, where $k(\lambda)$ is the dust extinction law. Adopting the Calzetti (1997) extinction law, $k\left(\left[\mathrm{O}_{\mathrm{II}}\right]\right)=5.86, k(\mathrm{H} \beta)=4.60, k\left(\left[\mathrm{O}_{\mathrm{III}}\right]\right)=4.46$, and $k(\mathrm{H} \alpha)=3.33$. This exercise enables us to estimate the oxygen abundance using the $R_{23}$ index based on extinctioncorrected line ratios. Based on the calibration of Yin et al. $(2007), 12+\log (\mathrm{O} / \mathrm{H})=6.486+1.401 \times \log R_{23}$, which is justified by the upper limit of the $N 2$ index, we find $12+\log (\mathrm{O} / \mathrm{H})=7.7,7.5$, and $7.6^{5}$ for the galaxies with increasing $d$ (see column 11 of Table 7 ). Uncertainties in the gas phase metallicity are driven by the systematic uncertainty of the $R_{23}$ calibration and it is estimated to be 0.1 dex (Yin et al. 2007).

While robust emission-line fluxes have been obtained for all three dwarf galaxies, accurate broad-band photometry is only feasible for the two galaxies at $d=37$ and 150 pkpc, not affected by the glare of the QSO. The observed $r$-band magnitudes of these two galaxies are $A B(r)=25.1$ and 24.1, respectively. We estimate an intrinsic $r$-band absolute magnitude $M_{r}$ using a star-forming galaxy template and find $M_{r}=-16.3$ and -17.3 for the two galaxies, corresponding to 0.01 and $0.025 L_{*}$ adopting $M_{r_{*}}=-21.3$ for blue galaxies from Cool et al. (2012). We further estimate the underlying stellar mass $M_{\text {star }}$ using the prescription for blue galaxies presented in Johnson et al. (2015), and find $\log M_{\text {star }} / \mathrm{M}_{\odot}=7.8$ and 8.2 , respectively. Both galaxies are found to be exceedingly faint and low-mass. Based on the estimated ISM gas phase metallicity of $\approx 10 \%$ solar and adopting the mass-metallicity relation of dwarf galaxies (e.g., Berg et al. 2012), we argue that the closest galaxy at $d=15 \mathrm{pkpc}$ is also likely to be a low-mass dwarf of $\log M_{\text {star }} / \mathrm{M}_{\odot} \approx 8$. The observed and derived properties of these three galaxies are summarized in columns (2) through (11) of Table 7.

\section{APPENDIX C: PROPERTIES OF A ROTATING DISK GALAXY AT $D=67$ PKPC FROM THE LLS AT $Z_{\mathrm{ABS}}=0.4353$ TOWARD J0357-4812}

The optical spectrum of the absorbing galaxy is characterized by strong absorption features, together with weak $[\mathrm{O} \mathrm{II}]$ and $\mathrm{H} \beta$ emission lines, indicating that this is an earlytype galaxy (see $\S 4.5 .2$ and Figure 11). At $z=0.4353$, MUSE does not provide the spectral coverage necessary for observing $\mathrm{H} \alpha$, limiting our ability in obtaining robust constraints for the dust content, and consequently for the ISM gas-phase metallicity and ionization condition of the galaxy. However, the presence of the [O II] emission doublet does enable measurements of the velocity field across the galaxy as well as an estimate of the unobscured SFR. To determine an integrated $[\mathrm{OII}]$ line flux and the velocity centroid at each pixel, we fit the emission doublet using KUBEVIZ, visually inspect the fitting results across the full field, and repeat the

from Gordon et al. (2003) does not change the extinctioncorrected line ratios significantly.

5 For comparison, the Sun has $12+\log (\mathrm{O} / \mathrm{H}) \odot=8.69 \pm 0.05$ (Asplund et al. 2009). 
fitting procedure as needed after modifying the input parameters. We find a total integrated $[\mathrm{O} \mathrm{II}]$ line flux over the entire galaxy of $f_{[\mathrm{OII}]}=(6.14 \pm 0.06) \times 10^{-17} \mathrm{erg} \mathrm{s}^{-1} \mathrm{~cm}^{-2}$, leading to an unobscured SFR of $\approx 0.28 \mathrm{M}_{\odot} \mathrm{yr}^{-1}$ based on the star formation calibrator of Kewley et al. (2004).

The observed velocity gradient along the long axis in panel (b) of Figure 10 supports the presence of a rotating disk. To investigate the relative motion of the LLS with respect to the rotation of the galaxy, we first estimate the inclination and orientation of the underlying disk using the pseudo $r$-band image. The outline of the galaxy appears to be largely well-defined by an ellipse in the pseudo $r$ band image with a mild irregular feature toward the northwest corner, as suggested also by the [O II] emitting morphology. We find that the galaxy can be characterized by an inclination angle of $i \approx 44^{\circ}$ and a position angle of the major axis of $\alpha \approx 120^{\circ}$, north through east. If there exists an extended gaseous disk, then the QSO sightline probes the gas at $\approx 27^{\circ}$ from the major axis. Next, we de-project both the projected distance $d$ and $d v_{\text {gal }}$ observed along the major axis onto the disk plane. Following the prescription described in Chen et al. (2005), the galactocentric radius $R$ is related to $d$ according to $R=$ $d \sqrt{1+\sin ^{2}(\phi-\alpha) \tan ^{2}(i)}$, while the de-projected velocity is related to the observed line-of-sight velocity according to $\Delta v_{\text {deproj }}=\Delta v \sqrt{1+\sin ^{2}(\phi-\alpha) \tan ^{2}(i)} / \cos (\phi-\alpha) / \sin (i)$, where $\phi$ is the position angle of the slit. For the galaxy disk, the MUSE data cube enables us to place a pseudo slit along the major axis, in which case $\phi_{\text {gal }}=\alpha$, whereas for the LLS $\phi$ is dictated by the location of the QSO probe relative to the galaxy and we find $\phi_{\mathrm{QSO}} \approx 93^{\circ}$.

The de-projected rotation velocity along the disk plane as a function of galactocentric radius in panel (c) of Figure 10 also includes the three strongest $\mathrm{H}_{\mathrm{I}}$ absorbing components (c1, c2, and c3 from Table 6) of the LLS along the QSO sightline for comparison (open circles in panel $c$ of Figure 10). Under the assumption that the gas is moving along the plane extended from the inclined optical disk, the zero velocity in panel $(c)$ of Figure 10 corresponds to the systemic redshift of the galaxy. While the rotation curve appears to flatten at $d v_{\text {deproj }} \approx+225 \mathrm{~km} \mathrm{~s}^{-1}$ on the east side of the galaxy, it continues to extend beyond $\left|d v_{\text {deproj }}\right| \approx 300$ $\mathrm{km} \mathrm{s}^{-1}$ on the west side which is likely related to the extended structure revealed in [O II] emission. 